\title{
Polarized NIR and X-ray flares from Sagittarius $A^{*}$
}

\author{
A. Eckart ${ }^{1,2}$, F. K. Baganoff ${ }^{3}$, M. Zamaninasab ${ }^{1,2}$, M. R. Morris ${ }^{4}$, R. Schödel ${ }^{1}$, L. Meyer ${ }^{1,2}$, K. Muzic ${ }^{1,2}$, M. W. Bautz ${ }^{3}$, \\ W. N. Brandt ${ }^{5}$, G. P. Garmire ${ }^{5}$, G. R. Ricker ${ }^{3}$, D. Kunneriath ${ }^{1,2}$, C. Straubmeier ${ }^{1}$, W. Duschl ${ }^{6}$, M. Dovciak ${ }^{7}$, V. Karas ${ }^{7}$, \\ S. Markoff ${ }^{8}$, F. Najarro ${ }^{9}$, J. Mauerhan ${ }^{4}$, J. Moultaka ${ }^{10}$, and A. Zensus ${ }^{2,1}$
}

1 I. Physikalisches Institut, Universität zu Köln, Zülpicher Str.77, 50937 Köln, Germany e-mail: eckart@ph1.uni-koeln.de

2 Max-Planck-Institut für Radioastronomie, Auf dem Hügel 69, 53121 Bonn, Germany

3 Center for Space Research, Massachusetts Institute of Technology, Cambridge, MA 02139-4307, USA

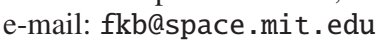

4 Department of Physics and Astronomy, University of California Los Angeles, Los Angeles, CA 90095-1562, USA

5 Department of Astronomy and Astrophysics, Pennsylvania State University, University Park, PA 16802-6305, USA

${ }^{6}$ Institut für Theoretische Physik und Astrophysik Christian-Albrechts-Universität zu Kiel Leibnizstr. 1524118 Kiel, Germany

7 Astronomical Institute, Academy of Sciences, Boční II, 14131 Prague, Czech Republic

8 Astronomical Institute "Anton Pannekoek", University of Amsterdam, Kruislaan 403, 1098SJ Amsterdam, The Netherlands

9 Instituto de Estructura de la Materia, Consejo Superior de Investigaciones Científicas, CSIC, Serrano 121, 28006 Madrid, Spain

${ }^{10}$ Observatoire Midi-Pyrénées, 14 avenue Édouard Belin, 31400 Toulouse, France

Received 4 October 2007 / Accepted 6 December 2007

\section{ABSTRACT}

\begin{abstract}
Context. Stellar dynamics indicate the presence of a supermassive 3-4 $\times 10^{6} M_{\odot}$ black hole at the Galactic Center. It is associated with the variable radio, near-infrared, and X-ray source Sagittarius A* (SgrA*).

Aims. The goal is the investigation and understanding of the physical processes responsible for the variable emission from SgrA*. Methods. The observations have been carried out using the NACO adaptive optics (AO) instrument at the European Southern Observatory's Very Large Telescope (July 2005, May 2007) and the ACIS-I instrument aboard the Chandra X-ray Observatory (July 2005)

Results. We find that for the July 2005 flare the variable and polarized NIR emission of SgrA* occurred synchronous with a moderately bright flare event in the X-ray domain with an excess $2-8 \mathrm{keV}$ luminosity of about $8 \times 10^{33} \mathrm{erg} / \mathrm{s}$. We find no time lag between the flare events in the two wavelength bands with a lower limit of $\leq 10 \mathrm{~min}$. The May 2007 flare shows the highest sub-flare to flare contrast observed until now. It provides evidence for a variation in the profile of consecutive sub-flares.

Conclusions. We confirm that highly variable and NIR polarized flare emission is non-thermal and that there exists a class of synchronous NIR/X-ray flares. We find that the flaring state can be explained via the synchrotron self-Compton (SSC) process involving up-scattered X-rays from the compact source component. The observations can be interpreted in a model involving a temporary disk with a short jet. In the disk component the flux density variations can be explained by spots on relativistic orbits around the central supermassive black hole (SMBH). The profile variations for the May 2007 flare can be interpreted as a variation of the spot structure due to differential rotation within the disk.
\end{abstract}

Key words. black hole physics - X-rays: general - infrared: general - accretion, accretion disks - Galaxy: center - Galaxy: nucleus

\section{Introduction}

At the center of our Galaxy at a distance of only $\sim 8 \mathrm{kpc}$ a supermassive black hole $(\mathrm{SMBH})$ of mass $\sim 4 \times 10^{6} M_{\odot}$ can convincingly be identified with the compact radio and infrared source Sagittarius A* (SgrA*) (Eckart \& Genzel 1996; Genzel et al. 1997, 2000; Ghez et al. 1998, 2000, 2003, 2005; Eckart et al. 2002; Schödel et al. 2002, 2003; Eisenhauer 2003, 2005; Reid \& Brunthaler 2004). Due to its proximity SgrA* provides us with the unique opportunity to understand, the physics and possibly the evolution of supermassive black holes at the nuclei of galaxies. Although, for a black hole of its size, SgrA* is extremely under luminous at about $10^{-9 \ldots-10} L_{\mathrm{Edd}} \mathrm{SgrA}^{*}$ is also the source of variable emission in the X-ray and nearinfrared wavelength regime (Baganoff et al. 2001, 2003; Eckart et al. 2004, 2006a; Porquet et al. 2003; Goldwurm et al. 2003;
Genzel et al. 2003b; Ghez et al. 2004; Eisenhauer et al. 2005; Belanger et al. 2006; and Yusef-Zadeh et al. 2006a). The NIR/X-ray variability is probably also linked to the variability at radio through sub-millimeter wavelengths showing that variations occur on time scales from hours to years (e.g. Bower et al. 2002; Herrnstein et al. 2004; Zhao et al. 2003, 2004; Markoff et al. 2007, 2005; Mauerhan et al. 2005, and references therein). The surprisingly low luminosity has motivated many theoretical and observational efforts to explain the processes that are at work in the immediate vicinity of SgrA*. For a recent summary of accretion models and variable accretion of stellar winds onto SgrA* see Yuan (2006) and Cuadra \& Nayakshin (2006).

The temporal correlation between rapid variability of the near-infrared (NIR) and X-ray emission (Eckart et al. 2004, 2006a) suggests that the emission showing $10^{33-34} \mathrm{erg} / \mathrm{s}$ flares 
Table 1. Observation log.

\begin{tabular}{cccll}
\hline \hline $\begin{array}{c}\text { Telescope } \\
\text { Observing ID }\end{array}$ & Instrument & Energy/ $\lambda$ & $\begin{array}{l}\text { UT and JD } \\
\text { Start Time }\end{array}$ & $\begin{array}{l}\text { UT and JD } \\
\text { Stop Time }\end{array}$ \\
\hline VLT UT 4 & NACO & $2.2 \mu \mathrm{m}$ & 2007 15 May 05:29:00 & 15 July 09:42:00 \\
& & \multirow{2}{*}{$2.2 \mu \mathrm{m}$ 2 454 235.72847 } & JD 2 454 235.90417 \\
VLT UT 4 & \multirow{2}{*}{ NACO } & \multirow{2}{*}{ 2005 30 July 02:04:00 } & 30 July 03:34:00 \\
& & JD 2 453 581.58611 & JD 2 453 581.64861 \\
Chandra & \multirow{2}{*}{ ACIS-I } & \multirow{2}{*}{$2-8 \mathrm{keV}$} & 2005 29 July 19:52:55 & 30 July 09:07:36 \\
& & & JD 2 453 581.32841 & JD 2 453 581.88028 \\
\hline
\end{tabular}

arises from a compact source within a few tens of Schwarzschild radii of the SMBH. For SgrA* we assume $R_{\mathrm{s}}=2 R_{\mathrm{g}}=2 G M / \mathrm{c}^{2} \sim$ $8 \mu$ as, with $R_{\mathrm{S}}$ being one Schwarzschild radius and $R_{\mathrm{g}}$ the gravitational radius of the SMBH. Until now for several simultaneous flare events the investigators found no time lag larger than an upper limit of $\leq 10 \mathrm{~min}$, mainly given by the required binning width of the X-ray data. The flaring state can be explained with a synchrotron self-Compton (SSC) model involving up-scattered sub-millimeter photons from a compact source component. Inverse Compton scattering of the THz-peaked flare spectrum by the relativistic electrons then accounts for the X-ray emission. This model allows for NIR flux density contributions from both the synchrotron and SSC mechanisms. Indications for red (Eisenhauer et al. 2005; Hornstein et al. 2006; Gillessen et al. 2006) and variable NIR spectra (Gillessen et al. 2006) is indicative of a possible exponential cutoff of the NIR/MIR synchrotron spectrum (Eckart et al. 2006a).

There is also evidence for a $17 \pm 3$ min quasi-periodic modulation of the NIR and X-ray emission (Genzel et al. 2003b; Eckart et al. 2006b; Meyer et al. 2006a,b; Belanger et al. 2006; Aschenbach et al. 2004a,b). In the following we refer to this phenomenon as QPO: quasi periodic oscillation. The NIR flare emission is polarized with a well defined range over which the position angle of the polarized emission is changing $\left(60^{\circ} \pm 20^{\circ}\right.$; Eckart et al. 2006b; Meyer et al. 2006a,b, 2007). All these observations can be explained in a model of a temporary accretion disk harboring a bright orbiting spot possibly in conjunction with a short jet (Eckart et al. 2006b; Meyer et al. 2006a,b, 2007), suggesting a stable orientation of the source geometry over the past few years.

The millimeter/submillimeter wavelength polarization of SgrA* is variable in both magnitude and position angle on timescales down to a few hours. Marrone et al. (2007) present simultaneous observations made with the Submillimeter Array polarimeter at 230 and $350 \mathrm{GHz}$ with sufficient sensitivity to determine the polarization and rotation measure at each band. From their measurements they deduce an accretion rate that does not vary by more than $25 \%$ and - depending on the equipartition constraints and the magnetic field configuration - amounts to $2 \times 10^{-5}$ to $2 \times 10^{-7} M_{\odot} \mathrm{yr}^{-1}$. The mean intrinsic position angle is $167^{\circ} \pm 7^{\circ}$ with variations of $\sim 31^{\circ}$ that must originate in the sub-millimeter photosphere of SgrA*.

Here we present new X-ray measurements that we obtained using Chandra and that were taken in parallel with the NIR polarization measurements reported by Eckart et al. (2006b). We also present new NIR polarization measurements taken in May 2007. In Sect. 2 we summarize the observations and the data reduction. The observational results and modeling of the data are presented in Sect. 3 and a more general discussion of available infrared and X-ray variability data on $\mathrm{SgrA}^{*}$ is given in Sect. 4. In the Appendix we outline the used synchrotron self Compton (SSC) mechanism and a multi-component model for the SgrA* temporal accretion disk. The results are summarized in Sect. 5 and conclusions are drawn.

\section{Observations and data reduction}

As part of a large observing campaign SgrA* was observed in May 2007 and July 2005, using the VLT ${ }^{1}$. In July 2005 we carried out simultaneously observations, using the Chandra X-ray observatory (hereafter Chandra). In the following we describe the data acquisition and reduction for the individual telescopes. Details of the observations are summarized in Table 1.

\subsection{NIR observations and data reduction}

The observations of SgrA* have been carried out in the NIR $K_{\mathrm{S}}$-band $(2.0-2.36 \mu \mathrm{m})$ using the NIR camera CONICA and the adaptive optics (AO) module NAOS on the European Southern Observatory's Very Large Telescope UT4 on Paranal, Chile, during the nights between 29 and 30 July 2005 as well as 14 and 15 May 2007. The infrared wavefront sensor of NAOS was used to lock the AO loop on the NIR bright ( $K$-band magnitude $~ 6.5$ ) supergiant IRS 7, located about 5.6" north of SgrA*. Therefore the $\mathrm{AO}$ was able to provide a stable correction with a high Strehl ratio (of the order 50\%). In NACOS/CONICA (NACO) the combination of a Wollaston prism with a half-wave retarder plate allows the simultaneous measurement of two orthogonal directions of the electric field vector.

Eckart et al. (2006b) report that the variable NIR emission of SgrA* observed in July 2005 is highly polarized and consists of a contribution of a non- or weakly polarized main flare with highly polarized sub-flares, showing a possible QPO of $17 \pm$ 3 min consistent with previous observations. Significant, positive flux density excursions on time scales shorter (according to all previous NACO observations typically on the QPO time scale) than the duration of the overall flare (typically 100 min or more) are called sub-flares. All further details of the observations and data reduction as well as an interpretation of the July 2005 data in the framework of an orbiting spot model are given in Eckart et al. (2006b) and Meyer et al. (2006b).

For the $K$-band polarimetry in May 2007 presented here, we took a total of 146 frames alternating between the $0^{\circ} / 90^{\circ}$ and $45^{\circ} / 135^{\circ}$ settings. For each image we used 4 sub-images $(\mathrm{NDIT}=4)$ at a $10 \mathrm{~s}$ exposure time (DIT $=10 \mathrm{~s})$. The images were corrected for bad pixels, sky, and flat field. The point spread function was extracted on each individual image (Diolaiti et al. 2000) and then used for a Lucy-Richard deconvolution. After restoration with a Gaussian beam, aperture photometry was done on the diffraction limited images for individual sources with known flux and SgrA*. For the extinction correction we assumed

\footnotetext{
1 Based on observations at the Very Large Telescope (VLT) of the European Southern Observatory (ESO) on Paranal in Chile; Programs: 073.B-0775 July 2004; 075.B-0093 July 2005; 079.B-0084 May 2007.
} 


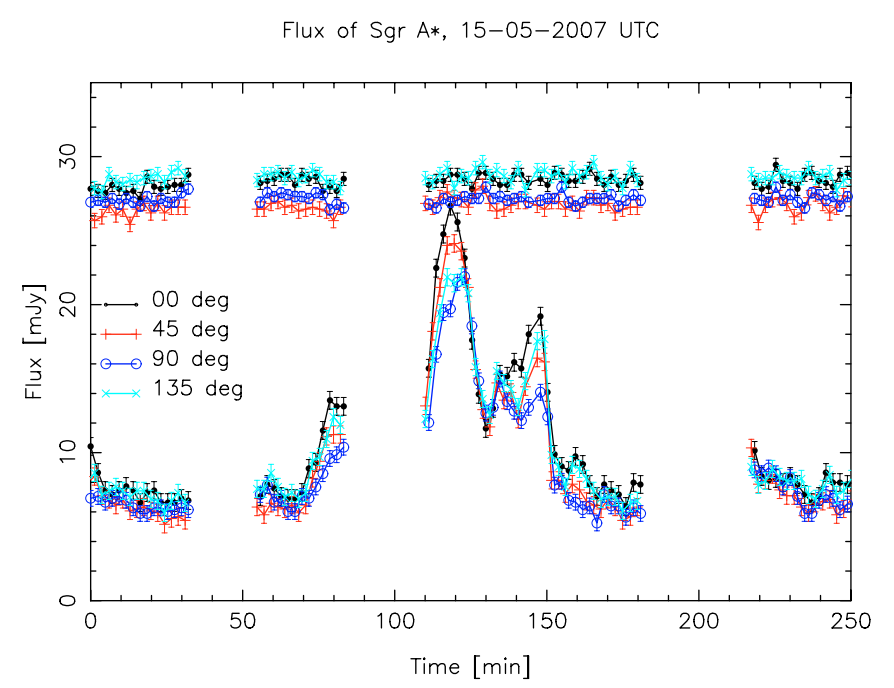

Fig. 1. The flux of the observed flare of SgrA* on 15 May 2007 in different channels as a function of time - each channel depicted in different color. Start and stop times are listed in Table 1. The light curve of a constant star, S2 (23 mJy at $2.2 \mu \mathrm{m}$, with $\left.A_{K}=2.8\right)$ is shown in the same plot and shifted by a few mJy for a better view.

$A_{K}=2.8$ mag. Estimates of uncertainties were obtained from the standard deviation of fluxes from nearby constant sources. The calibration was performed using the overall interstellar polarization of all sources in the field, which is $4 \%$ at $25^{\circ}$ (Eckart et al. 1995; Ott et al. 1999).

In Figs. 1 and 2 we show the flux densities per polarization channel, the total intensity, degree of polarization and the polarization angle as a function of time. The dereddened flux of SgrA* and of a nearby constant star are shown in the top panel of Fig. 1. The flux was calibrated such that each angle separately matches the total flux of known,, presumably intrinsically unpolarized stellar sources. That means that actually Fig. 1 shows approximately twice the flux for each angle. The 3 gaps in the data are due to sky observations.

The May 2007 flare shows 2 bright sub-flares centered, about 120 and $140 \mathrm{~min}$. The time difference between them is $20 \mathrm{~min}$ which is fully consistent, with the possible QPO found for other polarized NIR flares of SgrA* showing a sub-flare structure. The flux density between the 2 sub-flares does not reach the emission level well before and after (i.e. $<70$ and $>170 \mathrm{~min}$ into the observations) the flare. This off-flare emission is most likely dominated by residual stellar flux density contributions, especially from a faint stellar source ( $\mathrm{S} 0-17)$ which is currently at an apparent separation of $\sim 0.1$ arcsec from the position of $\mathrm{SgrA}^{*}$, but still well separated from it. Based on this fact and in comparison to previously observed flares and sub-flares (Genzel et al. 2003b; Eckart et al. 2006b; Meyer et al. 2006a,b, 2007; Trippe et al. 2007) we assume that there is an underlying flare emission. The May 2007 flare shows the highest sub-flare to flare contrast observed until now:

$C=\left(S_{\max }-S_{\min }\right) / S_{\min } \sim 2: 1$.

Here $S_{\max }$ is a sub-flare flux density peak and $S_{\text {min }}$ the mean of the neighboring minima. The second sub-flare is broader than the first one and shows a flat or even double peaked profile at the top. In Sect. 3.1 we interpret these profile variations as a variation of the spot structure due to differential rotation within the disk. Furthermore the flare is characterized by a very broad minimum in the degree of polarization, which is preceded by the
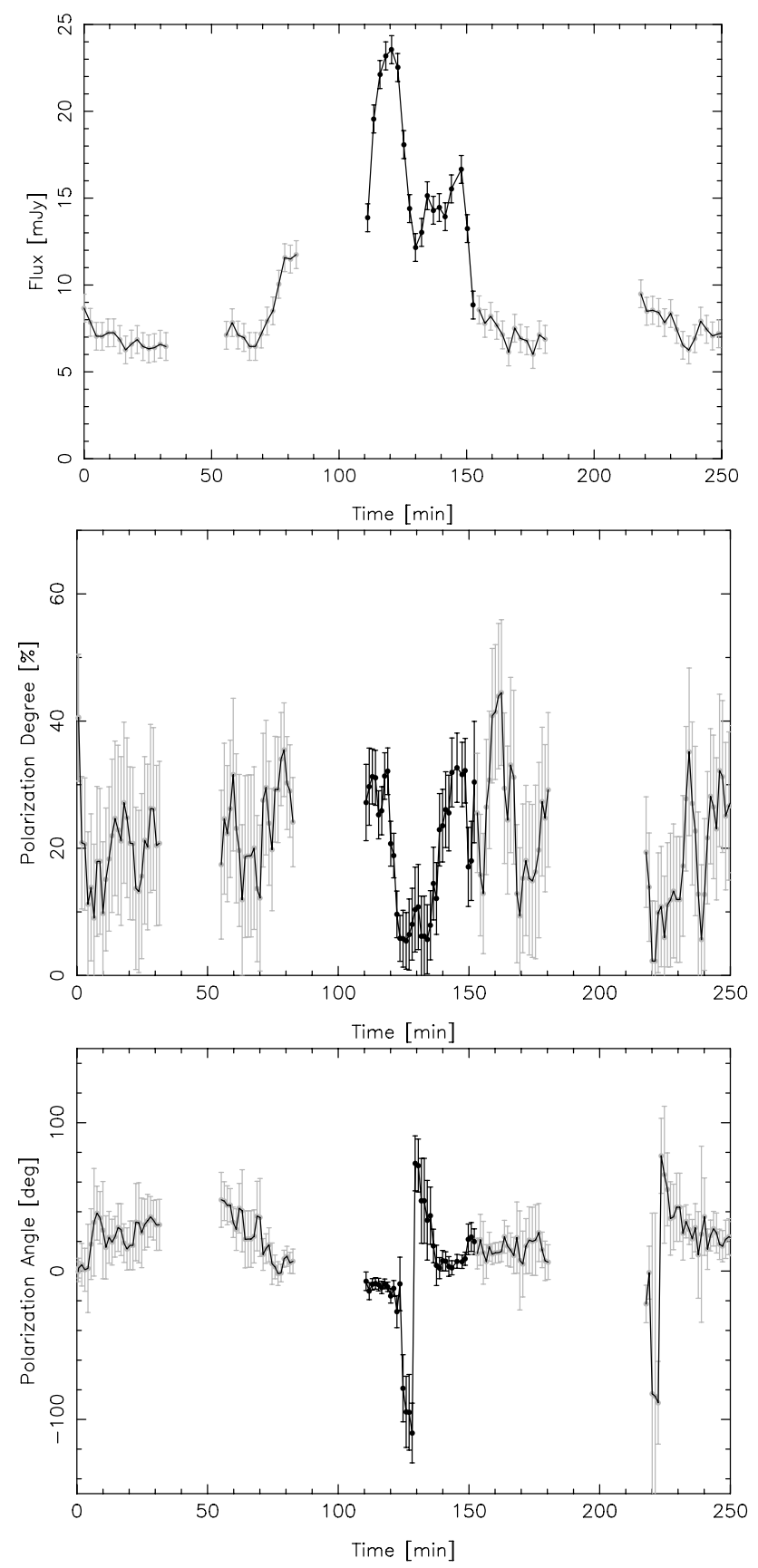

Fig. 2. The SgrA* NIR flare observed on 15 May 2007. Start and stop times are listed in Table 1. As a function of time we show the total flux density, degree of polarization and polarization angle of the $\boldsymbol{E}$-vector. The otherwise grey error bars are plotted in black during the time of the largest flux excursions due to the flare.

peak of the brightest sub-flare. During this minimum the position angle is not very well determined. However, at the center of the minimum and allowing for a possible phase wrap of $180^{\circ}$, the trend the polarization angle shows towards the center of the minimum is consistent with the value of $60^{\circ} \pm 20^{\circ}$, a value reported during the bright flare phase for all other flare events (see discussion in Meyer et al. 2007).

\subsection{The Chandra $X$-ray observations}

In parallel to the NIR observations (Eckart et al. 2006b; see below), SgrA* was observed with Chandra using the imaging 
Table 2. Here we list data of the flares observed by the VLT and Chandra. X-ray flare data: given are the start and stop times and peak ACIS-I count rates in $10^{-3} \mathrm{cts} \mathrm{s}^{-1}$ and $10^{-9}$ Jansky in $2-8 \mathrm{keV}$ band of the total flare emission and the flare emission corrected for the count rate during the quiescent state. We also list the estimated start and stop times, the full width at zero power $(F W Z P)$ and full width at half maximum $(F H W M)$ values, as well as the peak and quiescent flux densities. NIR flare data are given as for the X-ray flare. The peak flare flux densities are given in $10^{-3}$ Jansky in the NIR $K_{\mathrm{s}}$-band.

\begin{tabular}{llcccccccc}
\hline \hline $\begin{array}{l}\text { spectral } \\
\text { domain }\end{array}$ & Date & Flare Start & Flare Stop & $\begin{array}{c}F W Z P \\
(\mathrm{~min})\end{array}$ & $\begin{array}{c}F W H M \\
(\mathrm{~min})\end{array}$ & $\begin{array}{c}\text { Extraction } \\
\text { Radius } \\
(\operatorname{arcsec})\end{array}$ & Total & $\begin{array}{c}\text { Flare } \\
\text { state }\end{array}$ & $\begin{array}{c}\text { quiescent } \\
\text { NIR }\end{array}$ \\
& 15 May 2007 & $7: 30 \pm 5 \mathrm{~min}$ & $8: 30 \pm 5 \mathrm{~min}$ & $100 \pm 10$ & $>40$ & 0.06 & $16 \pm 0.5 \mathrm{mJy}$ & $16 \pm 0.5 \mathrm{mJy}$ & - \\
NIR & 30 Jul. 2005 & $<02: 04$ & $>03: 34$ & $>90$ & $70 \pm 10$ & 0.06 & $8 \pm 0.5 \mathrm{mJy}$ & $8 \pm 0.5 \mathrm{mJy}$ & - \\
X-ray & 30 Jul. 2005 & $01: 59 \pm 12 \mathrm{~min}$ & $04: 07 \pm 7 \mathrm{~min}$ & $128 \pm 19$ & $64 \pm 10$ & 1.50 & $25 \pm 3$ & $18 \pm 3$ & $7 \pm 1$ \\
& & & & & & & $70 \pm 8 \mathrm{nJy}$ & $51 \pm 8 \mathrm{nJy}$ & $20 \pm 3 \mathrm{nJy}$ \\
\hline
\end{tabular}

array of the Advanced CCD Imaging Spectrometer (ACIS-I; Weisskopf et al. 2002) for $\sim 50 \mathrm{ks}$ on 29-30 July 2005 in the 2-8 keV band. The start and stop times are listed in Table 1 . The instrument was operated in timed exposure mode with detectors I0-3 turned on. The time between CCD frames was $3.141 \mathrm{~s}$. The event data were telemetered in faint format.

We reduced and analyzed the data using CIAO v2.32 software with Chandra CALDB v2.22 $2^{3}$. Following Baganoff et al. (2003), we reprocessed the level 1 data to remove the $0.25^{\prime \prime}$ randomization of event positions applied during standard pipeline processing and to retain events flagged as possible cosmic-ray after-glows, since the strong diffuse emission in the Galactic Center causes the algorithm to flag a significant fraction of genuine X-rays. The data were filtered on the standard ASCA grades. The background was stable throughout the observation, and there were no gaps in the telemetry.

The X-ray and optical positions of three Tycho-2 sources were correlated (Høg 2000) to register the ACIS field on the Hipparcos coordinate frame to an accuracy of $0.10^{\prime \prime}$ (on axis); we then measured the position of the X-ray source at SgrA* ${ }^{*}$. The X-ray position $\left[\alpha_{\mathrm{J} 2000.0}=17^{\mathrm{h}} 45^{\mathrm{m}} 40.030^{\mathrm{s}}, \delta_{\mathrm{J} 2000.0}=\right.$ $\left.-29^{\circ} 00^{\prime} 28.23^{\prime \prime}\right]$ is consistent with the radio position of SgrA* (Reid et al. 1999) to within $0.18^{\prime \prime} \pm 0.18^{\prime \prime}(1 \sigma)$.

Extracting the counts from $\operatorname{SgrA} *$ in the $2-8 \mathrm{keV}$ band in an 1.0" aperture provides the best compromise between maximizing source signal and rejecting background (Baganoff et al. 2001, 2003; Eckart et al. 2004, 2006a). Background counts were extracted from an annulus around $\mathrm{SgrA}^{*}$ with inner and outer radii of $2^{\prime \prime}$ and $10^{\prime \prime}$, respectively, excluding regions around discrete sources and bright structures (Baganoff et al. 2003). The mean (total) count rates within the inner radius subdivided into the peak count rates during a flare and the corresponding quiescent values are listed in Table 2. The background rates have been scaled to the area of the source region. We note that the mean source rate in the $1.5^{\prime \prime}$ aperture is consistent with the mean quiescent source rates from previous observations (Baganoff et al. 2001, 2003).

\subsection{Comparison of the NIR/X-ray flare events}

The Chandra X-ray data fully cover the polarized NIR flare that we observed at the VLT in July 2005. The X-ray data show a $8 \times 10^{33} \mathrm{erg} / \mathrm{s}$ flare that is about 3 times as bright as the quiescent emission from SgrA* (Table 2). In Fig. 3 we show the Xray data using a 207 and a $628 \mathrm{~s}$ bin size. The cross-correlation of the X-ray data with the flux densities in the individual NIR

\footnotetext{
${ }^{2}$ Chandra Interactive Analysis of Observations (CIAO),

http://cxc.harvard.edu/ciao

${ }^{3}$ http://cxc.harvard.edu/caldb
}

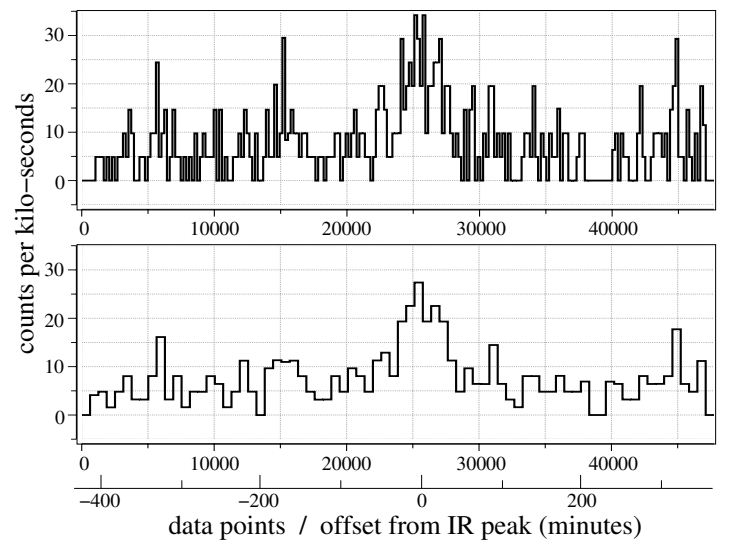

Fig. 3. The Chandra 2-8 keV X-ray data from 29/30 May 2005 shown in 207 and $628 \mathrm{~s}$ bins. The UT start and stop times are listed in Table 1.

polarization channels show that the flare event observed in the two wavelength bands is simultaneous to within less than $10 \mathrm{~min}$ (Figs. 4 and 5). The two sub-peaks in the cross-correlation function correspond to two apparent sub-peaks in the X-ray light curve that can, however, not be taken as significant given the $S N R$ of $\sim 3 \mathrm{cts} / \mathrm{s}$ per integration bin. In the X-ray domain there is no clear indication for a sub-flare structure as observed in the NIR. The NIR sub-flare to flare contrast (see definition above) ranges between $C=0.3$ and 0.9 (see Sect. A.1). For the NIR and $\mathrm{X}$-ray flare we list the start and stop times, flare widths, peak count rates and flux densities in Table 2.

\section{Modeling results}

\subsection{Relativistic disk modeling of the variable flare emission}

We interpret our polarized infrared flare events via the emission of spots on relativistic orbits around the central SMBH in a temporary disk (Eckart et al. 2006b; Meyer et al. 2006a,b, 2007). The model calculations are based on the KY-code by Dovciak et al. (2004) and are usually done for a single spot orbiting close to the corresponding last stable orbit. The KY-code takes special and general relativistic effects into account by using the concept of a transfer function (Cunningham 1975). The transfer function relates the flux as seen by a local observer comoving with an accretion disk to the flux as seen by an observer at infinity. This transfer of photons is numerically computed by integration of the geodesic equation. The possibility to explore effects of strong gravity via time-resolved polarimetric observations of X-rays was originally proposed by Connors \& Stark (1977). Two extreme cases of the intrinsic magnetic field configurations - purely poloidal and purely toroidal - were examined 


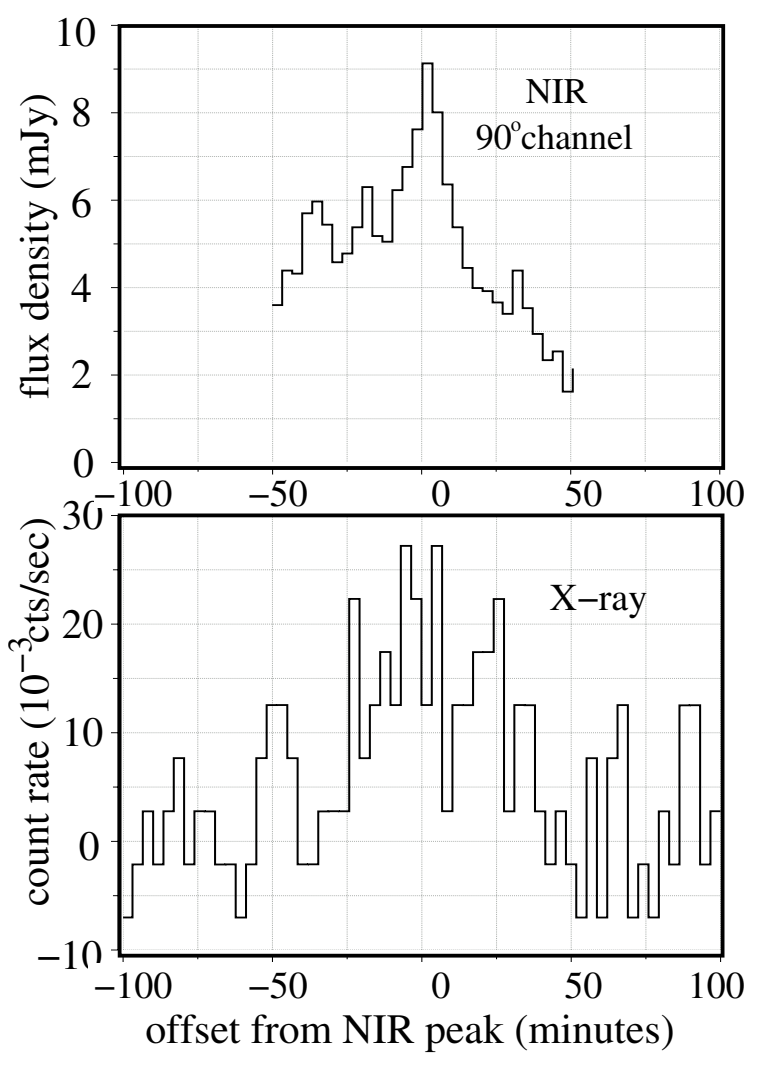

Fig. 4. The NIR (top) and X-ray (bottom) data for the SgrA* flare observed on 30 May 2005. To highlight both the flare and the sub-flare structure we plot the flux in the NIR $90^{\circ}$ polarization channel. Both data sets are shown in 207 bins. The X-ray data are corrected for the quiescent emission. The flare data are listed in Table 2. The peak of the NIR flare occurred at 02:56:00 UT $\pm 3 \mathrm{~min}$. To within about $\pm 7 \mathrm{~min}$ the X-ray peak time occurred at the same time.

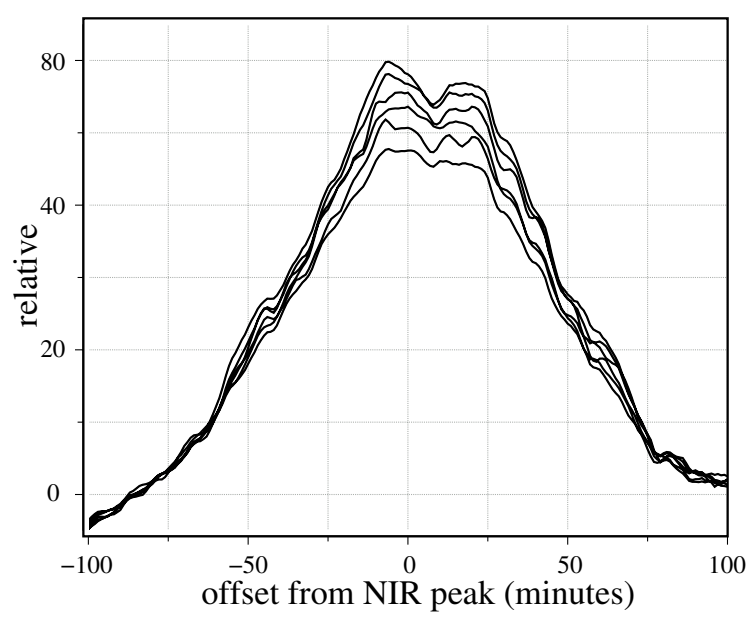

Fig. 5. Results of the cross correlation of the 207-s binned Chandra $\mathrm{X}$-ray data with the polarized emission seen at position angles of (top to bottom) $90^{\circ}, 120^{\circ}, 60^{\circ}, 150^{\circ}, 30^{\circ}, 00^{\circ}$.

as a toy model. Special and general relativistic effects (the relativistic beaming, redshifts and blue-shifts, lensing, time delays, the change of the emission angle and the change of polarization angle) in the polarized light near a Schwarzschild black hole (Pineault 1981) are calculated. This allows us to fit the model parameters to actual data. The procedure was first demonstrated
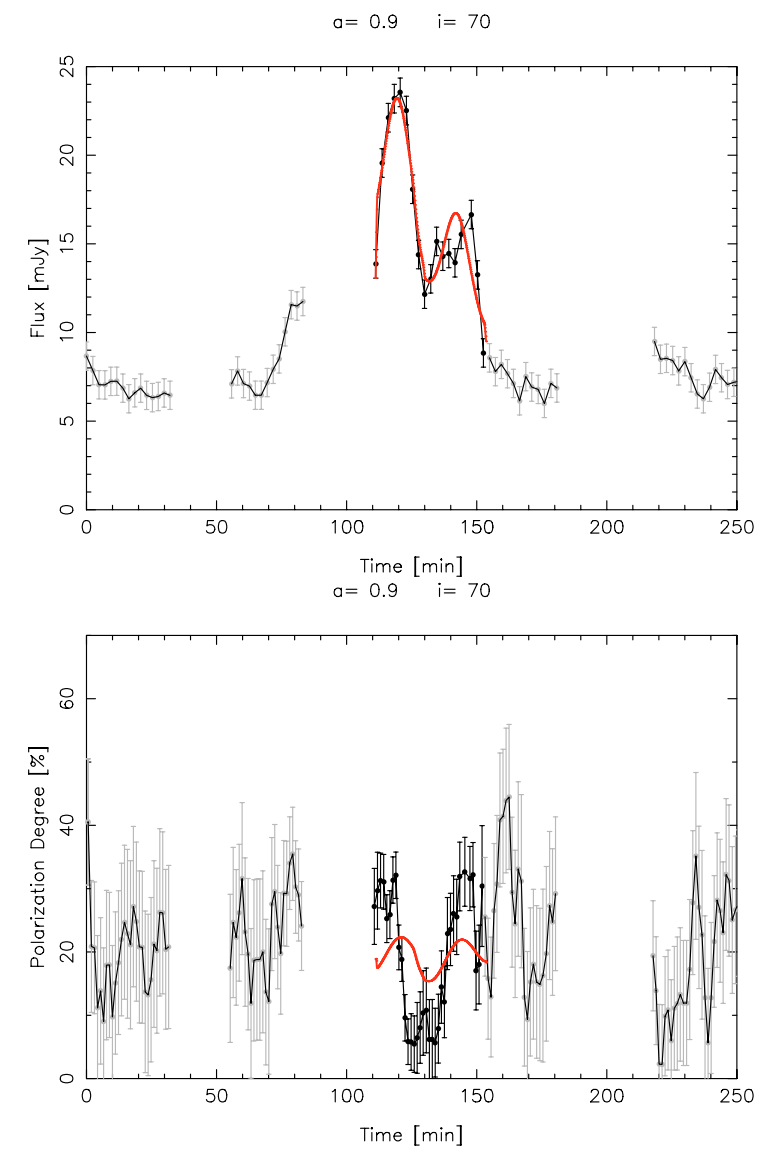

Fig. 6. The total flux and degree of polarization for a single spot during two revolutions for perpendicular E-field configuration. The otherwise grey error bars are plotted in black during the time of the largest flux excursions due to the flare. The parameters of the corresponding model (I) are given in Table 3.

by Meyer et al. (2006a,b; the authors also discuss in detail the differences with other modeling efforts, e.g. Broderick \& Loeb 2006a,b). The first configuration is such that the resulting projected $\boldsymbol{E}$-vector is always perpendicular to the equatorial plane (see also Shakura \& Sunyaev 1973). As a second configuration we have allowed for a global toroidal magnetic field (Hirose et al. 2004).

Here we report on how the May 2007 polarimetric observations may show a sign of evolution in the orbiting spot during the flare. Fitting the flare with the KY-code, we used only the total flux density and the degree of polarization, as the position angle of the $\boldsymbol{E}$-vector is not very well determined during the minimum in the degree of polarization.

Figure 6 shows the fit with the minimum reduced $\chi^{2}$-value for synchrotron radiation, from a highly polarized spot orbiting twice around the SMBH above the innermost stable circular orbit (ISCO). Since we have assumed that the spot mainly emits synchrotron radiation the intrinsic and global configuration of the magnetic field play an important role in the predictions by the model. Here we fitted the flux and intensity of polarization for the spin parameter $a$, the inclination angle $i$, an overall Gaussian shaped flare background, the over-brightness of the spot relative to the disk, polarization degree of the disk (restricted between 0-20\%) and the spot (restricted between 0-70\%) and the initial phase of the spot on the orbit. The inclination $i$ is defined such that the temporary accretion disk is seen edge-on at an inclination of $i=90^{\circ}$. The dimensionless spin parameter $a$ describes 
the spin of the massive black hole. A non-rotating black hole has a spin parameter $a=0$. A maximally spinning black hole has a spin parameter of $a=1$. The background upon which the subflare modulation is superimposed is centered at $105 \mathrm{~min}$ and has a $F W H M$ of $80 \mathrm{~min}$. The upper limit for the spot polarization reflects the maximum value that could be produced by synchrotron radiation. Figure 6 corresponds to a disk with a perpendicular $\boldsymbol{E}$-field configuration. The minimum reduced- $\chi^{2}$ value of 4.03 is achieved for a near extremal SMBH $(a=0.9)$ and a high inclination angle $\left(i=70^{\circ}\right)$. The $\chi^{2}$ values are calculated using the data points with the black error bars. Assuming that the errors are not under-estimated the fit quality, i.e. better $\chi^{2}$-values, is limited by our simple physical model. We had to assume a factor $f_{\mathrm{c}}=0.7$ by which the flux of the components has to decrease between the first and the second revolution. The physical origin of this factor is not clear, however, we assume it to be due to "cooling" of the spot via synchrotron losses. The model data are plotted as a continuous smooth (red) line. The amplitude of the model behaves similar to observed data but the degree of polarization and depolarization is not so high during the sub-flares. Within this scenario it is not possible to produce the shoulderlike sub-structure of the second peak. Also the rise and fall in the degree of the polarization at about 120 and $140 \mathrm{~min}$ are not sharp enough in comparison with the observations.

Within the framework of a (single) orbiting spot model, the timescale of the signal variations, the predicted contrast of the observed light-curve and the changes of polarization degree are constrained and mutually interconnected. As mentioned above, by assuming that the observed variations are dominated by the bulk orbital motion of the source, the timescales are given by the orbital radius and the black hole angular momentum, whereas the magnitude of the variations increases with the source inclination and reaches its maximum for the edge-on view. It appears that a single persisting spot is insufficient to reproduce the observed properties of the high contrast flare event. In agreement with the multi-component disk model presented in Sect. A we therefore applied another approach in which we employed a 2 spot model. This double spot approach can also be interpreted in an evolutionary framework, motivated by the fact that accretion disks show magneto-rotational instabilities in which magnetic field lines provide a coupling between disk sections at different radii resulting in an efficient outward transport of angular momentum. Inner disk portions that have lost angular momentum will then slide into lower lying orbits, and rotate yet more rapidly (Hawley \& Balbus 1991; Balbus 2003). Following this idea we model the effects of differential rotation simply by assuming two similar blobs with the same initial orbital phases but different radial positions within the range of typical spots (spot size radius $r_{\text {spot }} \sim 1-2 \quad R_{\mathrm{g}}$ ) that successfully described previous flares and sub-flares. Also we selected these two radii such that the mean orbital radius is about $4.8 R_{\mathrm{g}}$ according to the period of the flare and that the sub-flare structure is fitted by the overall model. Such a configuration explaining the flare activity of SgrA* can successfully simulate a single spot that evolves in time and separates into two entities after the first orbit.

Figure 7 clearly shows that this model can produce the substructures in flux and also the polarization degree. The fit prefers a high inclination angle and spin parameter $\left(i=70^{\circ}\right.$ and $a=0.7$; reduced $\chi^{2}=3.55$ ) which is also in good agreement with previous observations by Eckart et al. (2006b) and Meyer et al. (2006a,b). We used the same assumptions for the flare background as for the single spot model. The two spots orbit around the central SMBH for two revolutions with the same initial orbital phases but different radii $\left(\delta r=1.2 R_{\mathrm{g}}\right)$ for the case of the
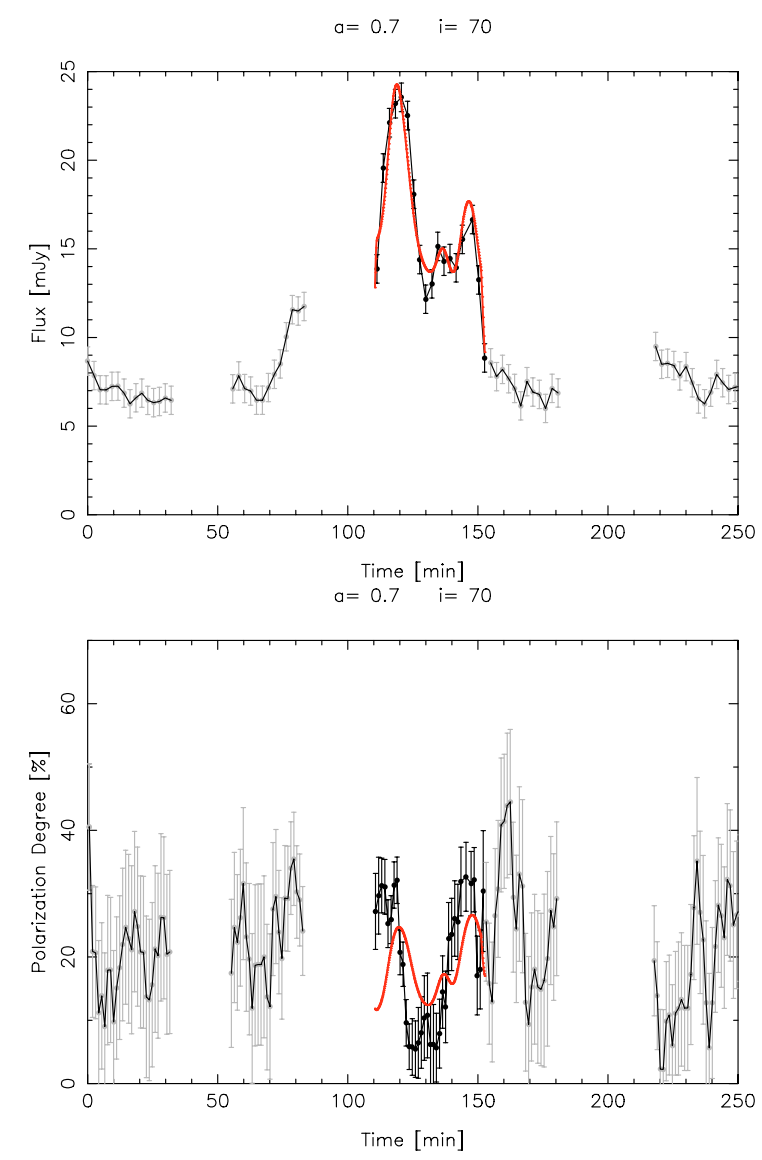

Fig. 7. The best fit that could be achieved by a double hot spot model for May 2007 data. The otherwise grey error bars are plotted in black during the time of the largest flux excursions due to the flare. The parameters of the corresponding model (II) are given in Table 3.

E-field perpendicular to the disk. As the spots separate completely from each other after the first orbit, this model reproduces the observed sharp rises and falls in flux and the degree of polarization.

Finally, in the case of an azimuthal magnetic field the results are quite similar to the findings reported by Meyer et al. $(2006 a, b)$. We find for the best fit curves a reduced $\chi^{2}$-value of 4.60. This field configuration therefore is not favored.

\section{A multi-component disk model}

The observed NIR/X-ray properties of the SgrA* light curves raise a number of questions: Can we expect a sub-flare structure in the X-ray domain using a synchrotron self Compton model? What is the approximate flux distribution within a temporary accretion disk around SgrA*? This is also closely related to more general questions (discussed in Sect. 4) of how the observed light curve properties vary if the lifetime of the spot, and the shearing and synchrotron cooling time scales are considered.

In a multi-component model for the temporal accretion disk we combine the light amplification curves for individual orbiting spots (based on the KY-code) and a simple SSC model. Thereby we obtain zero order time dependent flare characteristics from the NIR to the X-ray domain. We assume that the essential quantities of the SSC models, i.e. the turnover flux density $S_{\mathrm{m}}$, frequency $v_{\mathrm{m}}$, and the source size $\theta$ of the individual source components are distributed as power laws with the number distributions 
Table 3. Final fit parameters resulting from the relativistic modeling using the KY-code. The flux is given as the over-brightness ratio between the disk and the spot flux density at the location of the spot in the co-moving frame. The polarization degree $P$ is given for the spot and disk as well.

\begin{tabular}{ccccccc}
\hline \hline Model & Component & Flux & $\begin{array}{c}\text { Radius } \\
r \text { in } r_{\mathrm{g}}\end{array}$ & $\begin{array}{c}\text { Spot/disk } \\
P \text { in } \%\end{array}$ & $\begin{array}{c}\text { Spin parameter } \\
a\end{array}$ & $\begin{array}{c}\text { Inclination } \\
i\end{array}$ \\
\hline I. & 1 & 0.44 & 4.8 & $34 / 0$ & 0.9 & $70^{\circ}$ \\
& & & & & & \\
II. & 1 & 17.0 & 4.2 & $51 / 10$ & 0.7 & $70^{\circ}$ \\
& 2 & 17.0 & 5.4 & $51 / 10$ & 0.7 & $70^{\circ}$ \\
\hline
\end{tabular}
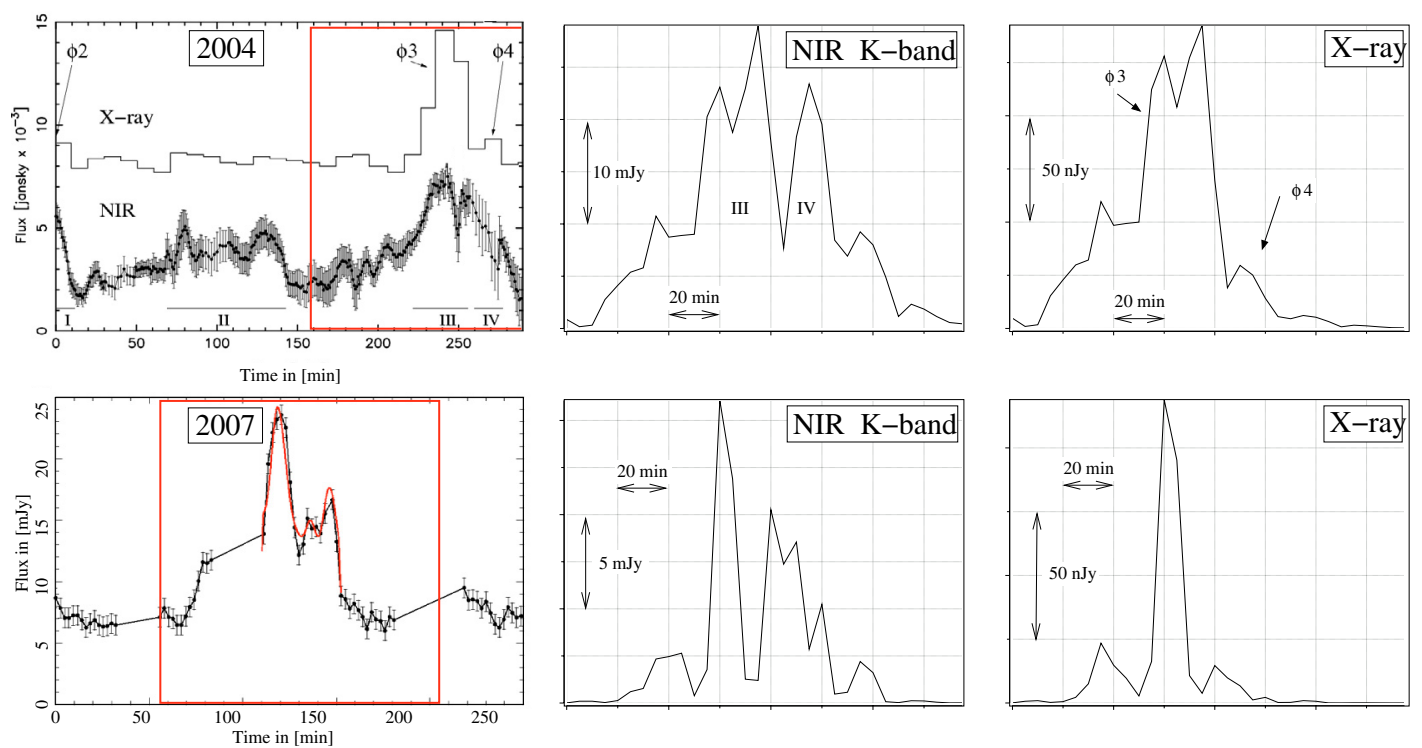

Fig. 8. Application of the time dependent flare emission model presented in Sect. A.2 to the data obtained in May 2007 (bottom) and July 2004 (top). In the panels on the left we show the available NIR and X-ray data with the modeled portion indicated by a red line. In the middle and on the right we show the NIR $K$-band and X-ray light curve derived from a multi-component disk model. In both cases, starting at the center of the flare event we assumed a 30\% increase of the source component sizes over about $40 \mathrm{~min}$ i.e. two orbital time scales. With the additional assumption of a flux decrease of $\sim 1$ mag due to synchrotron losses the model provides a very good qualitative and quantitative representation of the 2004 measurements (see Eckart et al. 2006a). For the 2007 NIR data there are no simultaneous X-ray data available but the $K$-band light curve indicates an evolution of the source components. The lower right panel therefore represents the light curve we would have expected.

of flux components within the temporal accretion disk

$$
N(S) \propto S_{\mathrm{m}}^{\alpha_{S}}, \quad N(v) \propto v_{\mathrm{m}}^{\alpha_{v}}, \quad N(\theta) \propto \theta^{\alpha_{\theta}} .
$$

In the Appendix we describe the details of this extended SSC model that allows us to describe the disk structure (including hot spots) and to calculate light curves in the NIR and X-ray domain in order to discuss the questions posed above.

We applied this multi-component disk model to the July 2005 and May 2007 observations presented here, as well as the July 2004 data presented by Eckart et al. (2006a; see Table A.2).

A comparison of the July 2005 X-ray data with noise sections added from the $207 \mathrm{~s}$ light curve in Fig. 3 to the noise free panels and the light curves shown in Fig. 4 demonstrates that at the given SNR and data sampling, QPOs in the X-ray data are difficult to determine, even if they have a modulation contrast similar to that observed in the NIR. Bright spots may on average have smaller sizes or lower cutoff frequencies. An increase of SSC X-ray flux density due to an increase of THz peak synchrotron flux may be compensated by this effect (see expressions by Marscher et al. 1983). Hence the sub-flare contrast may be much lower in the X-ray compared to the NIR domain.

In Fig. 8 we show the modeling results for the May 2007 NIR and the July 2004 simultaneous NIR/X-ray data on SgrA* using our time dependent flare emission model. For the 2007 data we implemented a double hot spot model as described in Sect. 3.1. For the 2004 data we invoke a model consisting of 7 components at increasing distances from the SMBH starting at the innermost stable orbit (see Appendix). The components line up on opposite sides of the SMBH close to the flare center in time, thus providing a maximum amount of Doppler amplification before and afterwards. This gives rise to the two NIR flare events labeled III and IV (Eckart et al. 2006a). Motivated by the fact that the May 2007 data shows evidence for a spot evolution due to differential rotation within the relativistic disk, we assumed that this may result in an increase of the source size for the individual spots as well. Therefore, starting at the center of the flare event, we assumed in both cases a $30 \%$ increase of the source component sizes over 30 to 40 min, i.e. about two orbital time scales. This results in a sharp decrease of the SSC $\mathrm{X}$-ray flux density and therefore in a very good representation of the 2004 measurements (see X-ray flares labeled $\phi 3$ and $\phi 4$ in Fig. 8 and Eckart et al. 2006a). Based on these time dependent model assumptions we would have expected a similarly strong evolution of the X-ray flare light curve for the May 2007 NIR observations as shown in the bottom right panel of Fig. 8.

Such a scenario may also explain the 2006 July 17 Keck NIR/X-ray light curves reported by Hornstein et al. (2007). The 
authors measured an NIR flare without a detectable X-ray counterpart. It was delayed by about $45 \mathrm{~min}$ from a significant X-ray flare, during which no NIR data was taken. Assuming that the $\mathrm{X}$-ray flare was accompanied by an unobserved NIR flare as well, this event may have been very similar in structure to our July 2004 flare.

\section{Discussion}

While the orbiting spot model is very successful in describing the suggested polarized quasi periodic near-infrared flares there are a few model assumptions that are worth being discussed (in Sect. 5.1) especially with respect to a growing body of observational flare data. To explain the feeble emission of SgrA* $\left(10^{-9 \ldots-11}\right.$ of the Eddington rate) an intense discussion among the theoretical community at present focuses on a combination of a radiatively inefficient accretion flow and jet models (e.g. Yuan et al. 2004; Narayan et al. 1995; Blandford \& Begelman 1979; Ball et al. 2001; Markoff et al. 2001, 2007, 2005). In Sect. 5.2 we discuss our results in the framework of a jet model. It is also of interest to outline links between possible accretion disk and wind/jet models which is done in Sect. 5.3.

\subsection{The orbiting spot model}

Large scale disk instabilities may be responsible for the overall flare lengths (Tagger \& Melia 2006; Meyer et al. 2006a). The light curves for the orbiting spot model have been calculated under the assumption that the spot remains confined, i.e. to first order preserved in strength and extent, for several orbital periods. Here the new May 2007 data possibly represents first direct observational evidence that spots may only be stable for about one orbital time scale. An efficient creation of spots could be provided through magneto-rotational instabilities that are shown to be present in Keplerian rotating accretion disks even in the presence of a dominating toroidal magnetic field (Hawley \& Balbus 1991; Blokland et al. 2005). Such shear-flow instabilities are a fast mechanism to generate a turbulent flow in a Keplerian disk (e.g. three-dimensional simulations by Arlt \& Rüdiger 2001). Differential rotation as well as the radial transport phenomena within the disk stretch out the magnetic field lines that may link two disk elements. This mechanism may provide the opportunity for field reconnection to occur. The magnetic loops connecting the two disk elements result in poloidal fields and may represent the spot (Hawley \& Balbus 1991). A spot lifetime that exceeds the theoretically predicted maximum of about one orbit (Schnittman 2005; Schnittman et al. 2006; see discussion in Sect. 5.2.2) appears to be indicated. In the following we have assumed that in each flare the sub-flares are dominated by the flux density contribution of a single spot.

\subsubsection{Spot stability within the disk}

If the spot is not confined it will be disintegrated via shear in the differentially rotating disk within a very few orbital periods. The synchrotron cooling time at $2.2 \mu \mathrm{m}$ is only a few minutes, i.e. significantly shorter than the time over which the spot may stay confined in the presence of shearing. If no further heating occurs this cooling time scale will dominate the time over which the spot persists. The synchrotron cooling time scale $t_{\mathrm{S}}$ can be calculated via

$t_{\mathrm{s}, \text { upper }}<3 \times 10^{7} \Gamma \delta^{0.5} v_{9}^{-0.5} B^{-3 / 2}$,

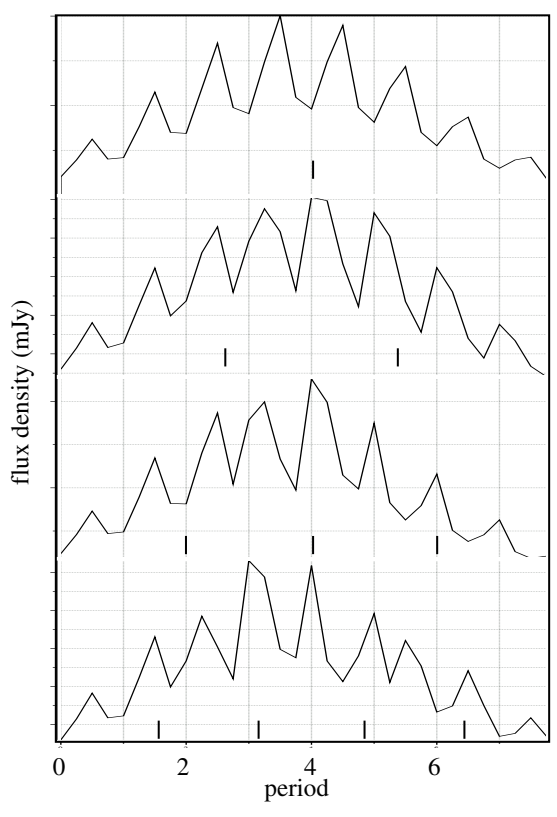

Fig. 9. Simulation of $2.2 \mu \mathrm{m}$ light curves under the assumption of decreasing times scale for the stability of source components in the accretion disk around SgrA*. The thin vertical lines mark the centers of Gaussian shaped stability time intervals. These marks are spaced by a $F W H M$ of the individual distributions. For short stability times scales the overall appearance of the light curve is preserved but the sub-flare amplitudes and time separations vary.

where $t_{\mathrm{s}}$ is in seconds, $B$ is in Gauss, $v_{9}$ is frequency in $\mathrm{GHz}$, and $\Gamma$ and $\delta$ are the relativistic factors for the bulk motion of the material (Blandford \& Königl 1979). If we adopt $B \sim 60 \mathrm{G}$ and $v_{9}=300-1600 \mathrm{GHz}$ with $\Gamma \sim \delta \sim 1.5$ as typical flare characteristics (see Eckart et al. 2006a) then the synchrotron cooling time of the THz-peaked overall flare emission is of the order of 1 to $2 \mathrm{~h}$ and matches the typical length of a flare event as observed in the $K$-band as well as the $2.5 \mathrm{~h}$ time scale found at $3 \mathrm{~mm}$ wavelength by Mauerhan et al. (2005). At $2.2 \mu \mathrm{m}$ we find a much shorter cooling time scale of $T_{\mathrm{S}} \approx 4.3 \mathrm{~min}$.

Combining the cooling time scale with the expression for the upper frequency of the synchrotron spectrum $v_{2}=2.8 \times 10^{6} B \gamma_{2}^{2}$ in $\mathrm{GHz}$ and setting it equal to the observing frequency $v_{9}$ we find that

$t_{\mathrm{s}} \propto v_{9}^{-2}$

This implies that the lifetime of a source component bright in the $L$-band at $3.8 \mu \mathrm{m}$ is $\approx 13 \mathrm{~min}$, three times longer than a component seen at $2.2 \mu \mathrm{m}$. At the same time the upper frequency of the synchrotron spectrum is a strong function of the peak synchrotron cutoff frequency and the source size. Due to $B \propto \theta^{4} v_{\mathrm{m}}^{5} S_{\mathrm{m}}^{-2}$ (e.g. Marscher 1983) we find:

$v_{2} \propto B \propto \theta^{4} v_{\mathrm{m}}^{5}$

Small variations in these quantities will result in a large variation of the upper synchrotron cutoff frequency and hence in a significant variation of the infrared flux density (and potentially the infrared spectral index; see Eckart et al. 2006a).

For Fig. 9 we calculated $2.2 \mu \mathrm{m}$ light curves under the assumption of decreasing times scale for the magneto-hydrodynamical stability of source components in the accretion disk 
around SgrA*. The thin vertical lines mark the centers of stability intervals with Gaussian shaped flux density weights. These marks are spaced by a FWHM of the individual Gaussians that result via modulation with the amplification curves in light curves similar to the observed ones. We assumed that for each of these intervals the flux density distribution within the disk is different. This results in phase shifts between the light curves (i.e. different positions of the spot within the disk) of $\pm \pi$. This simulation shows that the overall appearance (especially the mean QPO frequency) of the light curve can be preserved and that variations in the sub-flare amplitude and time separations can be explained by such a scenario. In Fig. 9 both quantities vary by a factor of 2. Larger variations are possible for stronger variations of the spot brightness.

In a simple model by Schnittman (2005) hot spots are created and destroyed around a single radius with random phases and exponentially distributed lifetimes $T_{\text {lif }}$, resulting in Lorentzian peaks in the power spectrum at the orbital frequency with a width of $\Delta v=\left(4 \pi T_{\text {lif }}\right)^{-1}$. The typical lifetimes of spots in this model are proportional to the orbital period $T_{\text {orb }}$ at their radius. From MHD calculations Schnittman et al. (2006) find over a large range of radii that disk perturbations indeed have a nearly exponential distribution of lifetimes, with $T_{\text {lif }} \sim 0.3 T_{\text {orb }}$. This implies that even if the spot lifetime is solely determined by the cooling time at 2.2 or $3.8 \mu \mathrm{m}$, this scenario is in full agreement with the suggested quasi-periodicity since $T_{\text {lif }} \sim 0.3 T_{\text {orb }} \sim T_{\mathrm{S}}$ (see above and Appendix).

Assuming that the width of the observed QPO is $17 \pm 3$ min we can derive an expected full width of the power spectrum peak of $\Delta v \sim 0.02 \mathrm{~min}^{-1}$. Following Schnittman et al. (2005) this corresponds to an expected lifetime of the spots of $T_{\text {lif }} \sim 4 \mathrm{~min}-\mathrm{a}$ value similar to the synchrotron cooling time in the NIR $K$-band. However, quasi-simultaneous $K$ - and $L$-band measurements by Hornstein et al. (2007) show that for several 1 to $2 \mathrm{~h}$ stretches of variable $K$-band emission $\geq 3 \mathrm{mJy}$, including flares of 10 to 30 min duration, the light curves at both wavelengths are well correlated. This suggests that the synchrotron cooling time scale in this case is not a relevant quantity for the spot stability. In addition the spread $\Delta v$ is an upper limit to the width of a possible Lorentzian distribution describing the QPO measurements. Therefore, we have to assume that $T_{\text {lif }}$ is even longer than the synchrotron cooling times at $K$ - and $L$-band, i.e. significantly longer than $13 \mathrm{~min}$, and suggesting that the spot lifetime could be of the order of $T_{\text {orb }}$, in agreement with results by Schnittman et al. (2005) and the model calculations presented here. The synchrotron cooling time scales may not be relevant at $K$ - and $L$-band if the heating time scale is longer (e.g. on the time scale of the overall NIR or sub-mm flare event) or if some additional mechanism is at work that stabilizes the spots in the temporary accretion disk of SgrA*. A small spot size and a high magnetic field intrinsic to the spot may help to prevent strong shearing, lowering the requirements on this confinement mechanism.

\subsection{The jet model}

Although in several cases NIR observations provide indications for QPOs ( $K$-band: Genzel et al. 2003b; Eckart et al. 2006b; Meyer et al. 2006a,b; as well as in a recent $L$-band light curve: Trippe 2007), they are not apparent in all NIR light curves (e.g. see $L$-band light curve by Hornstein et al. 2007). In addition, the radio to X-ray properties of SgrA* are often explained by emission from a jet (see e.g. Markoff et al. 2007, 2005), a source structure which is associated with almost all galactic

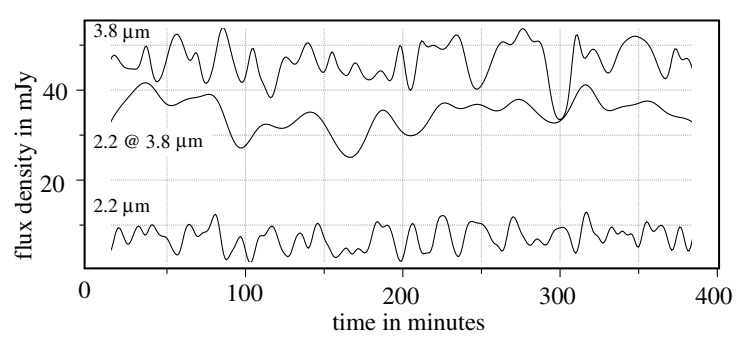

Fig. 10. Simulated light curves at $2.2 \mu \mathrm{m}$ and $3.8 \mu \mathrm{m}$ wavelength. The time scale has been set such that the rate of positive flux density excursions matches approximately the variability observed at $2.2 \mu \mathrm{m}$. The smooth central line shows the contribution of the $2.2 \mu \mathrm{m}$ events at $3.8 \mu \mathrm{m}$. All additional flux density variations seen at $3.8 \mu \mathrm{m}$ are due to lower energy components radiating longward of $2.2 \mu \mathrm{m}$.

nuclei. Therefore we discuss the polarization and variability data also in the framework of a possible jet model.

\subsubsection{The polarization angle}

The orientation of the polarization angle may be linked to the intrinsic source structure. In particular in the case of a jet there may be a preferred orientation of the E-vector relative to the jet orientation. Pollack et al. (2003) find for a sample of 177 sources a flat distribution of position angles with a tendency of the $\boldsymbol{E}$-vector being perpendicular to the jet direction. Rusk (1988) and Gabuzda \& Cawthorne (2000) find a weak indication for parallel orientations in stronger beamed jet sources. The mean 230 and $345 \mathrm{GHz}$ intrinsic position angle is $167^{\circ} \pm 7^{\circ}(\mathrm{E}$ of $\mathrm{N})$ with variations of $\sim 31^{\circ}$ (Marrone et al. 2007). Within the error this position angle is orthogonal to that of the NIR polarized emission at about $60-80^{\circ}(\mathrm{E}$ of $\mathrm{N})$. Both position angles also show a similar amount of variability (about $30^{\circ}$; Eckart et al. 2006b; Meyer et al. 2006a,b). Alternating orthogonal polarization angles are a common feature observed in jets. It is also very likely that the flare spectrum of $\mathrm{SgrA}^{*}$ is THz-peaked. In this case orthogonal polarization angles between frequencies above (NIR) and below ( $\mathrm{mm} / \mathrm{sub}-\mathrm{mm}$ domain) the synchrotron cutoff frequency are expected. However, the model by Liu et al. (2007) also explains the millimeter and sub-millimeter polarization properties with the emission originating entirely from a hot accretion disk. This supports that the emission is from small regions and therefore associated with flare events occurring either in coronae of the disk or within the last stable orbit. It also shows that the polarization angle itself is not a sufficient indicator to decide between a jet and disk structure.

\subsubsection{Variability and synchrotron cooling}

If the emission is originates in the foot point of a jet, we would expect that the time dependency of the flux density is not necessarily inferred via the amplification curves of the orbiting spot model but solely from jet instabilities and the synchrotron cooling. Here we investigate the effect of the the synchrotron cooling and show in Fig. 10 simulated simultaneous light curves at $2.2 \mu \mathrm{m}$ and $3.8 \mu \mathrm{m}$. For a mean magnetic field of $B \sim 60 \mathrm{G}$ we took the frequency dependence of the synchrotron cooling time into account. The light curve was calculated using the SSC formalism described in the Appendix resulting in source components with different upper synchrotron cutoff frequencies. We also assumed that the synchrotron heating time is shorter than the cooling time and that at any time the NIR/MIR spectrum is 


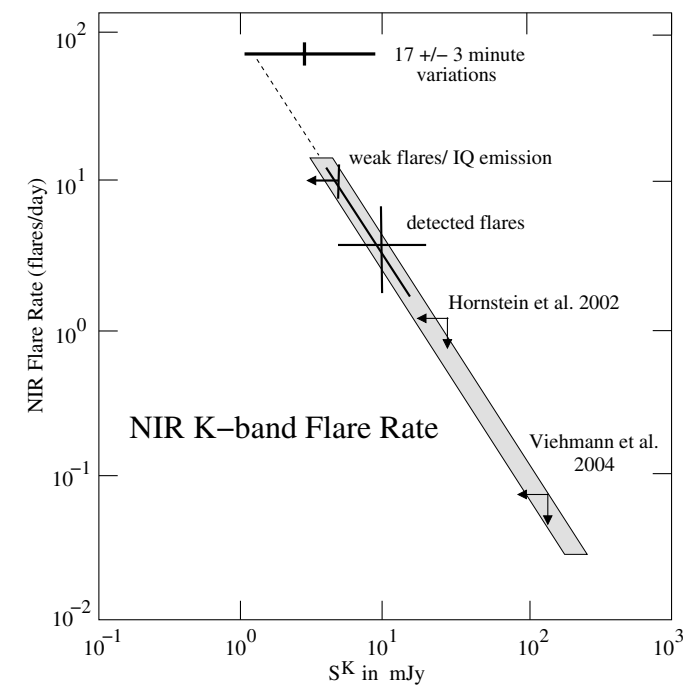

Fig. 11. Flare rate as a function of flare amplitude for the NIR $K$-band emission from SgrA* under the assumption that the characteristic flare duration is of the order of $100 \mathrm{~min}$ (see Eckart et al. 2006a). The $17 \pm$ 3 min variations observed as the sub-flare structure lies close to the extrapolation of the power law line derived from flare measurements. It includes all NIR flare events (see references in Sect. 5.2) as well as a $K$-band event with high sub-flare contrast observed in May 2007.

dominated by a single source component. Source components that are bright at $\leq 2.2 \mu \mathrm{m}$ are bright at $3.8 \mu \mathrm{m}$ for a correspondingly longer time. For source components that only appear at $3.8 \mu \mathrm{m}$ we assumed a minimum cooling time of $3 \mathrm{~min}$.

The middle curve in Fig. 10 (labeled $2.2 \mu \mathrm{m} @ 3.8 \mu \mathrm{m}$ ) shows the $3.8 \mu \mathrm{m}$ flux density contribution of the components that are bright at $2.2 \mu \mathrm{m}$ and shorter. All additional flux density contributions that are included in the $3.8 \mu \mathrm{m}$ light curve are due to source components with synchrotron cutoffs at wavelengths longward of $2.4 \mu \mathrm{m}$. Figure 10 demonstrates that, if the synchrotron cooling time is the only effect that is responsible for the observed flux density variations, then the light curves at longer infrared wavelengths will be strongly influenced by all lower energy synchrotron events and will not at all resemble the light curves observed at shorter NIR wavelengths. The quasi-simultaneous $K$ and $L$-band measurements by Hornstein et al. (2007) show that the $K$ - and $L$-band light curves at both wavelengths are well correlated, suggesting that the synchrotron cooling time scale in this case is not relevant. This implies that the heating time scale was longer or the component responsible for the flare event was stabilized by some mechanism (see Sect. 5.1.1).

\subsubsection{The $K$-band flare rate}

In case of a jet, the $17 \pm 3$ min infrared (optically thin) flux density variations may more likely be a result of the variations in the accretion process (or jet instabilities) rather than being a result of a modulation from an orbiting spot. In this case one may expect that red noise variations on these short times scales are a natural extension of the variability found for longer periods. Figure 11 shows the $\mathrm{SgrA}^{*}$ flare amplitude as a function of the flare rate at $2.2 \mu \mathrm{m}$ under the assumption that the characteristic flare duration is of the order of $100 \mathrm{~min}$ (see Eckart et al. 2006a). Longer average flare durations will shift the graph towards lower rates and vice versa. The $17 \pm 3$ min flux density variations lie close to the extrapolation of the power law line derived from flare measurements. However, while the sub-flare variability appears to be a

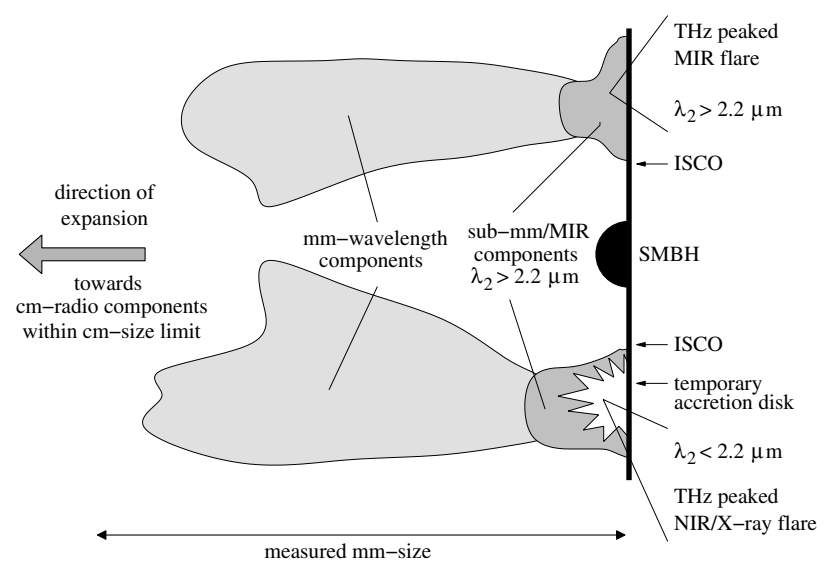

Fig. 12. Sketch of a possible source structure for the accretion disk around the SMBH associated with SgrA*. The disk is shown as a vertical thick line to the right. Extending to the left we show one side above the disk. Higher energy flare emission (lower part) is responsible for the observed NIR/X-ray flare emission. Lower energy flare emission (upper part) may substantially contribute to long wavelength infrared emission. In addition to the expansion towards and beyond the the $\mathrm{mm}$ source size, radial and azimuthal expansion within the disk may occur. Here $\lambda_{2}$ is the wavelength corresponding to the upper synchrotron cutoff frequency $v_{2}$.

natural extension of the flare rate spectrum, there is no evidence for a large number of $2.2 \mu \mathrm{m}$ flares with durations between 100 and $\sim 20 \mathrm{~min}$. The sub-flare variations also lie to the right of the flare rate power spectrum as it would be expected for any signal that is clearly discernable from the variations imposed by the flares. It therefore appears to be equally likely that the sub-flare variations are due to a separate mechanism and lie beyond the possible cutoff $\kappa_{2}$ for low flare amplitudes as discussed in Eckart et al. (2006a) or beyond an equally likely cutoff for high flare rates. Both cutoffs can be explained within the disk model proposed by Meyer et al. (2006a) in which the flare is due to a sound wave traveling within a finite disk. The disk size limitation may reflect itself in a typical flare duration and flux density as well as a possible drop in power for shorter variations. The higher sub-flare rates are then due to typical turbulence size scales of components within the disk (e.g. Hawley \& Balbus 1991; Arlt \& Rüdiger 2001). The brightest of these orbiting components would give rise to the observed QPOs. The finite orbiting disk may, however, be identical to the foot point of a jet or wind (see e.g. Markoff et al. 2007, 2005).

\subsection{A disk plus a short jet}

A source structure in which an accretion disk is associated with a short jet may explain most of the observed properties of SgrA*. Such a configuration is sketched in Fig. 12. In this figure the disk is seen edge-on. Details of expected jet geometries are discussed by Markoff et al. (2007). We show one side above the disk and two events that may be characteristic for higher and lower energy flare emission. The higher energy events would be responsible for the observed NIR/X-ray flares. The lower energy events would contribute most to long wavelength infrared emission. Higher energy events within the disk may be more stable and result in the observed sub-flare properties. Lower energy events may occur more detached from the disk, less stable and more dominated by the effects of synchrotron cooling (see however results by Hornstein et al. 2007). While the sub-mm emission will be intimately associated with the SSC flare events the 
mm-emission will originate after adiabatic expansion, further down stream the wind or jet emanating from the accretion disk. In addition in radial and azimuthal expansion of the emission zone within the disk may occur, which is consistent with the traveling sound wave picture presented by Meyer et al. (2006a). The sound wave or flare would be caused by the disk activity events depicted in Fig. 12. Further expansion of the wind or jet towards more extended and diffuse source components that dominate the cm-emission will occur.

A number of essential scenarii that comprise most of the properties associated with infrared/X-ray SgrA* light curves can be explained within the model:

- I. Let us assume that the mean upper synchrotron cutoff lies shortward of the NIR $K$-band, the source components are stable for several cooling time scales and the flux density variations are due to their orbital motion around SgrA*: in this case correlated QPOs should be observed in the NIR $K$ and $L$-bands. This situation corresponds to the orbiting spot model, represents events that take place within the accretion disk and will give rise to observed polarized infrared light curves that show quasi-periodic sub-flare structure.

- II. Here we assume that the mean upper synchrotron cutoff lies in or longward of the NIR $K$-band, but the flare events producing flux at increasingly longer wavelengths take place at increasing distances above the accretion plane: in this case QPOs are preferentially observed at short NIR wavelengths. At longer wavelengths they are less likely to occur and the variations are not strongly correlated with those at shorter NIR wavelengths. This scenario would be consistent with the presence of a short jet and would again be valid especially for lower energy events.

- III. The mean upper synchrotron cutoff lies shortward (but inconsistent with Hornstein et al. 2007) of the NIR $K$-band, but the source components are stable for only a few cooling time scales. In this case QPOs should be observed in e.g. the NIR $K$ - and $L$-bands, but they should be largely uncorrelated with respect to each other. This could especially be the case for lower energy events that result in infrared flux density variations but are not accompanied by significant X-ray flares.

- IV. If the source component flux density variations are dominated by the synchrotron cooling time scale and are not due to relativistic effects caused by the orbital motion of the components around $\mathrm{SgrA} *$ then the $K$ - and $L$-band light curves are not correlated with each other and no significant QPOs are observed. Such a scenario may be observed if the flux density variations occur above the disk, along the short jet or within the disk at larger disk radii.

The quasi-simultaneous $K$ - and $L$-band measurements by Hornstein et al. (2007) make cases III and IV less likely. Very weak X-ray events have also been reported by Eckart et al. (2006a). Here the events $\phi_{1}, \phi_{2}$ and $\phi_{4}$ could only be identified through infrared measurements. In these cases the X-ray peak flux density was only of the order of 1.2 to 1.8 times the quiescent X-ray flux density associated with SgrA*. Such weak X-ray events require that the constant bremsstrahlung and variable SSC component of SgrA* can be distinguished through sensitive, high angular resolution $\mathrm{X}$-ray measurements as provided by the ACIS-I instrument aboard the Chandra X-ray Observatory. However, we need to measure more flares to obtain a higher statistical significance.

If the entire NIR/X-ray flare event happens to occur above the $\operatorname{SgrA}^{*}$ accretion disk or extends a few $R_{\mathrm{g}}$ into the disk corona, then the modulation expected from an orbital spot may be significantly reduced. Above the disk the spot radiation will be subject to less gravitational bending from the black hole and potentially more extended. In that case a larger section of the underlying disk will be heated by the X-ray flare and an increasing amount of the lower infrared flare emission will be inverse Compton scattered rather than synchrotron self Compton scattered. These effects will also lead to a significant reduction of any QPO sub-flare contrast.

\section{Summary and conclusion}

We have measured a significant X-ray flare that occurred synchronous to a NIR flare with polarized sub-flares. This confirms the previous finding (Eckart et al. 2004, 2006a; Yusef-Zadeh et al. 2006a) that there exists a class of X-ray flares that show simultaneous NIR emission with time lags of less than $10 \mathrm{~min}$. In addition there are lower energy flare events that are bright in the infrared and are not detected in the X-ray domain (Hornstein et al. 2007). In the relativistic disk model the May 2007 polarimetric NIR measurements of a flare event with the highest subflare contrast observed until now, provides direct evidence for a spot evolution during the flare. This fact provides further strong support for the interpretation of the NIR polarimetry data within a relativistic disk model. Combined with the assumption of spot expansion due to differential rotation the combined SSC disk model can explain the July 2004 flare (Eckart et al. 2006a) and possibly also the 2006 July 17 flare reported by Hornstein et al. (2007).

The combination of relativistic amplification curves with a simple SSC mechanism allows us zero order interpretations in a time dependent flare emission model. We find that the temporary accretion disk around SgrA* can well be represented by a multi-component model with source properties that are bracketed by those of a simple flare and a quiescent model. We have used a $\left(\gamma_{\mathrm{e}} \sim 10^{3}\right)$ synchrotron model in which the source component spectral indices are compatible with the constant value of $\alpha=0.6 \pm 0.2$ reported by Hornstein et al. (2007). A steeper spectral index of $\alpha=1.3$ allows for direct synchrotron and SSC contributions in the NIR. In both cases the component flux densities can be represented by a power spectrum $N(S) \propto S_{\mathrm{m}}^{\alpha_{S}}$ with an exponent $\alpha_{S}$ close to -1 . The multicomponent model explains the quasi-periodic sub-flare structure at infrared wavelengths and shows that with adequate sensitivity and time resolution they should be detectable in the X-ray domain as well.

We also consider a model in which a combination of a temporary accretion disk occurs in combination with a short jet. This model can explain most of the properties associated with infrared/X-ray SgrA* light curves. Simultaneous NIR $K$ - and $L$-band measurements in combination with $\mathrm{X}$-ray observations should lead to a set of light curves that should allow us to prove the proposed model and to discriminate between the individual higher and lower energy flare events. Simultaneous X-ray measurements are important to clearly distinguish between high and low energy events. To do so it is required to separate the thermal non-variable bremsstrahlung and the non-thermal variable part of the SgrA* X-ray flux density. This capability is provided by the ACIS-I instrument aboard the Chandra X-ray Observatory and is essential to have, especially in the case of weak X-ray flare events in which the X-ray flare intensity is of the order of the extended bremsstrahlung component associated with SgrA* - or even below. These can clearly be identified in combination with infrared data. 
Acknowledgements. Part of this work was supported by the German Deutsche Forschungsgemeinschaft, DFG via grant SFB 494. L. Meyer, K. Muzic, M. Zamaninasab, and D. Kunneriath are members of the International Max Planck Research School (IMPRS) for Radio and Infrared Astronomy at the MPIfR and the Universities of Bonn and Cologne.

\section{Appendix A: The multi-component disk model}

In the following we describe an extended SSC model that includes a disk structure and allows us to calculate light curves in the NIR and X-ray domain in order to discuss the questions posed above.

\section{A.1. Description of the used SSC model}

Current models (Markoff et al. 2001; Yuan et al. 2002, 2003, 2004; Liu et al. 2006; Yuan 2006) predict that during a flare a few percent of the electrons near the event horizon of the central black hole are accelerated. These models give a description of the entire electromagnetic spectrum of SgrA* from the radio to the X-ray domain. In contrast to these global solutions, here we limit our analysis to modeling the NIR to X-ray spectrum of the most compact source component at the location of SgrA*. Our analysis is based on a simple SSC model describing the observed radio to X-ray properties of SgrA* using the nomenclature given by Gould (1979) and Marscher (1983). Inverse Compton scattering models provide an explanation for both the compact NIR and X-ray emission by up-scattering sub-mm-wavelength photons into these spectral domains. The models do not intend to explain the entire low frequency radio spectrum and quiescent state X-ray emission. However, they give a description of the compact emission from SgrA* during low and high flux density flare periods. A more detailed explanation is also given by Eckart et al. (2004, 2006a).

We assume a synchrotron source of angular extent $\theta$. The source size is of the order of a few Schwarzschild radii $R_{\mathrm{S}}=$ $2 G M / c^{2}$ with $R_{\mathrm{s}}=1.2 \times 10^{10} \mathrm{~m}$ for a $\sim 4 \times 10^{6} M_{\odot}$ black hole. One $R_{\mathrm{S}}$ then corresponds to an angular diameter of $\sim 8 \mu$ as at a distance to the Galactic Center of $\sim 8 \mathrm{kpc}$ (Reid 1993; Eisenhauer et al. 2003). The emitting source becomes optically thick at a frequency $v_{\mathrm{m}}$ with a flux density $S_{\mathrm{m}}$, and has an optically thin spectral index $\alpha$ following the law $S_{v} \propto v^{-\alpha}$. The upper synchrotron cutoff frequency is $v_{2}$. This allows us to calculate the magnetic field strength $B$ and the inverse Compton scattered flux density $S_{\mathrm{SSC}}$ as a function of the X-ray photon energy $E_{\mathrm{keV}}$ (Marscher 1983):

$S_{\mathrm{SSC}} \propto \ln \left(v_{2} / \nu_{\mathrm{m}}\right) \theta^{-2(2 \alpha+3)} \nu_{\mathrm{m}}^{-(3 \alpha+5)} S_{\mathrm{m}}^{2(\alpha+2)} E_{\mathrm{KeV}}^{-\alpha}$.

The synchrotron self-Compton spectrum has the same spectral index as the synchrotron spectrum that is up-scattered i.e. $S_{\mathrm{SSC}} \propto$ $E_{\mathrm{keV}}{ }^{-\alpha}$, and is valid within the limits $E_{\min }$ and $E_{\max }$ corresponding to the wavelengths $\lambda_{\max }$ and $\lambda_{\min }$ (see Marscher et al. 1983, for further details). We find that Lorentz factors $\gamma_{\mathrm{e}}$ for the emitting electrons of the order of typically $10^{3}$ are required to produce a sufficient SSC flux in the observed X-ray domain. A possible relativistic bulk motion of the emitting source results in a Doppler boosting factor $\delta=\Gamma^{-1}(1-\beta \cos \phi)^{-1}$. Here $\phi$ is the angle of the velocity vector to the line of sight, $\beta$ the velocity $v$ in units of the speed of light $c$, and Lorentz factor $\Gamma=\left(1-\beta^{2}\right)^{-1 / 2}$ for the bulk motion. With "bluk motion" we mean the collective motion of the emitting material of an entire source component with respect to the observer rather than the motions of the individual electrons. In the particular case of the hot spot model it is the bulk motion of individual sections of the accretion disk i.e. the orbiting spot. Relativistic bulk motion is not a necessity to produce sufficient SSC flux density but we have used modest values for $\Gamma=1.2-2$ and $\delta$ ranging between 1.3 and 2.0, representing a suitable coverage of the inclinations used in the models of the temporary accretion disk around SgrA* (see below). Such values will also be relevant in cases of mild relativistic outflows both of which are likely to be relevant to SgrA*.

With $\gamma_{\mathrm{e}} \sim 10^{3}$, the upper synchrotron cutoff frequency $v_{2}$ lies within or just short-ward of the NIR bands such that a considerable part of the NIR spectrum can be explained by synchrotron emission, and the X-ray emission by inverse Compton emission. This is supported by SSC models presented by Markoff et al. (2001) and Yuan et al. (2003) that result in a significant amount of direct synchrotron emission in the infrared (see also synchrotron models in Yuan et al. 2004; and discussion in Eckart et al. 2004).

\section{A.2. The SSC disk model}

In order to explain the time dependent flare properties we assume that the sub-flare and disk component can be described by a number of individual synchrotron and SSC emitting source components. Combining the light amplification curve for individual orbiting spots and the simple SSC model described above, we can obtain zero order time dependent flare characteristics from the NIR to the X-ray domain.

As a starting point we used synchrotron models that represent a high flux density, i.e. flaring, and a low flux density state. Greenhough et al. (2001) outline the importance of scaling properties of the transport processes operating within accretion disks. Pessah et al. (2007) present a scaling law between magnetic stress in units of the gas pressure and the vertical disk cell size in units of the pressure scale height implying that the magnetic field and source component size follow a power law relation. Therefore we assume that the essential quantities of the SSC models, i.e. the turnover flux density $S_{\mathrm{m}}$, frequency $v_{\mathrm{m}}$ and the source size $\theta$ of the individual source components are distributed as power laws with the boundary values taken from the high and low flux density state models. The exponents of the corresponding number distributions $N(S), N(v)$, and $N(\theta)$ of flux components within the temporal accretion disk are $\alpha_{S_{\mathrm{m}}}, \alpha_{v_{\mathrm{m}}}$ and $\alpha_{\theta}$ :

$N(S) \propto S_{\mathrm{m}}^{\alpha_{S}}, \quad N(v) \propto v_{\mathrm{m}}^{\alpha_{v}}, \quad N(\theta) \propto \theta^{\alpha_{\theta}}$.

For example if $\alpha_{S}=0$ the flux densities of the source components cover the full range between the minimum and maximum values. For $\alpha_{S}>0$ and $\alpha_{S}<0$ there is an increasing preference towards larger and lower flux density values, respectively. Similarly this is true for $\alpha_{v_{\mathrm{m}}}$ and $\alpha_{\theta}$.

The ISCO around a non-rotating black hole with spin parameter $a=0$ is $6 R_{\mathrm{g}}$. Assuming the co-rotating case that radius will shrink for higher spin parameters. For a rotating black hole with $a=0.5$ the radius is $\sim 4.4 R_{\mathrm{g}}$. Model calculations have shown (Meyer et al. 2006a,b) that for SgrA* spin parameters $a \geq 0.5$ and source components orbiting at radii larger than the ISCO are very likely. Meyer et al. (2006a) have shown that the disk is small with an outer disk radius extending not much further than $2 R_{\mathrm{S}}$ beyond the ISCO. With source components sizes of the order of $1.5 R_{\mathrm{g}}$ (Meyer et al. 2006a,b) we can therefore safely assume that the disk is well sampled using a total of 10 Gaussian shaped disk sections with random values of $S_{\mathrm{m}}, v_{\mathrm{m}}$ and $\theta$ taken from the described power law distributions in order to model the entire accretion disk. The brightest of these sections will then represent 

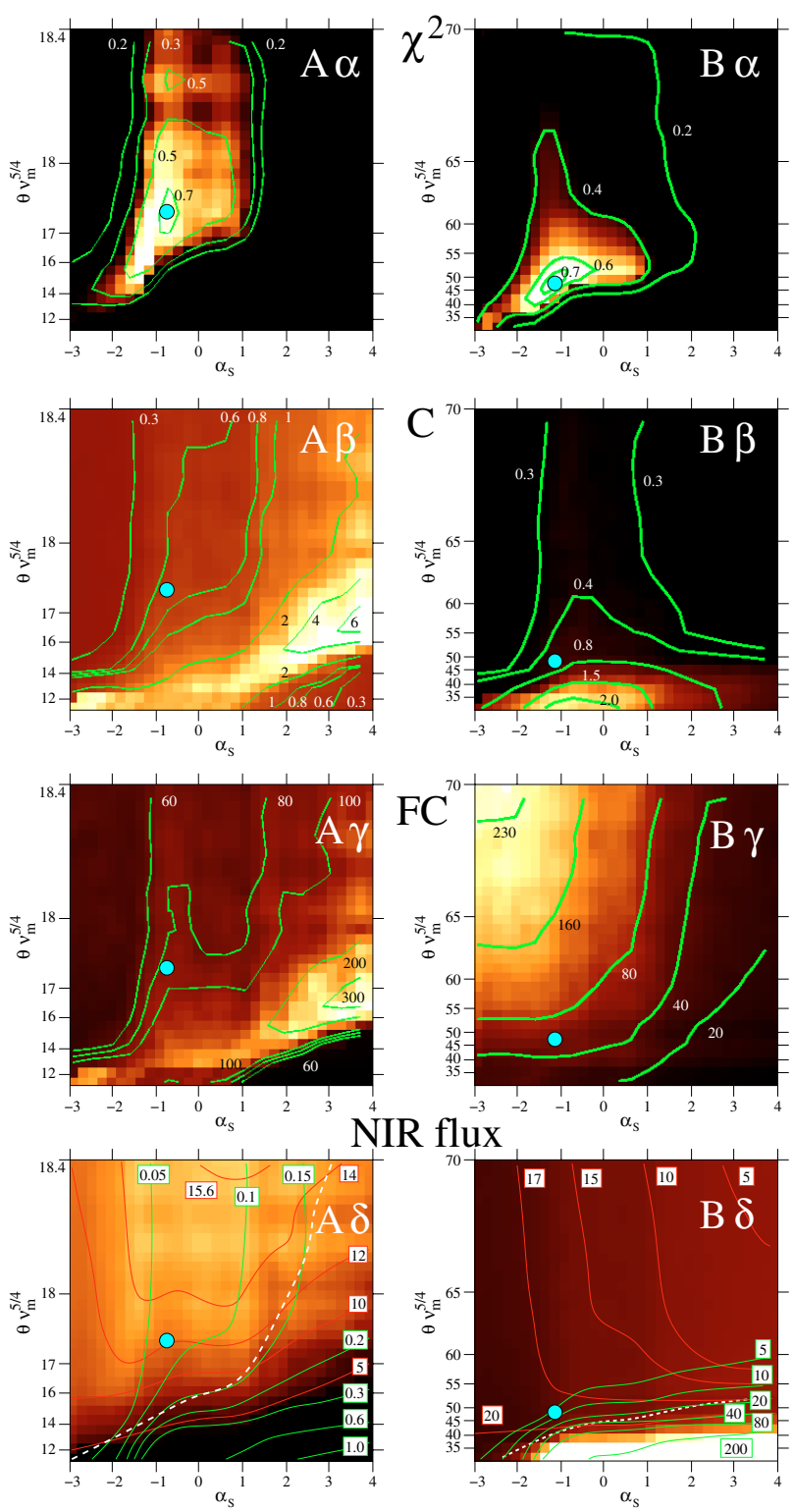

Fig. A.1. Diagnostic diagrams for two representative synchrotron models of the flare emission of SgrA*.

the orbiting spot and the rest will account for the underlying disk. This setup will of course also allow for several bright spots. This is shown for two cases in the top panels of Fig. A.2. As a simplebut still general - model we assumed the source components to be equally spaced along the circumference of a constant orbit. While orbiting, the flux density of each component will follow the achromatic magnification curves that can be calculated as a function of spin parameter $a$, inclination $i$ and orbital radius. In addition we infer a Gaussian shaped heightening function with a $F W H M$ of about 3 orbital periods, which resembles the observed flare lengths quite well.

In detail in Fig. A.2 the flux density distributions are shown along the last stable orbit perimeter of the supermassive black hole associated with $\operatorname{SgrA}^{*}$. Here no truncation at or just within the last stable orbit has been applied. We show the results for the synchrotron ( $A$, left panels) and synchrotron self Compton model ( $B$, right panels). The contour lines are at $12,25,50$, and $75 \%$ of the peak of the flux density distribution. The NIR and $\mathrm{X}$-ray light curves are representative for the median values at the
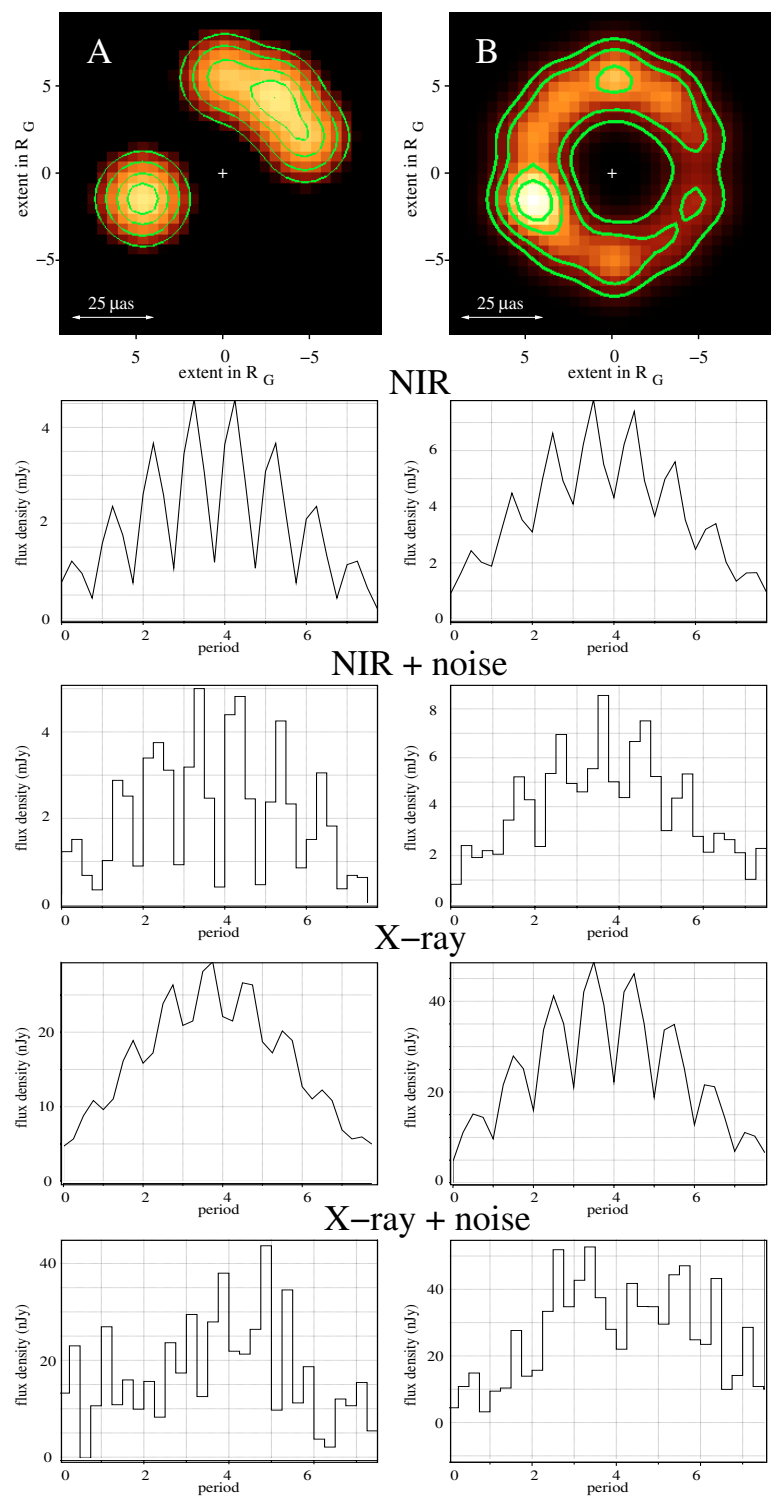

Fig. A.2. As results from the model calculations (see Fig. A.1) we show for two cases representative flux density distributions and NIR/X-ray light model curves with and without noise.

position of the $\chi^{2}$ minima in Fig. 4. For comparison we added sections of the 207 s light curve in Fig. 1 scaled to the peak values and bin size of the X-ray model light curves. For the NIR we added $0.4 \mathrm{mJy}$ of random Gaussian noise. The bin size of the model data corresponds to $207 \mathrm{~s}$ for a $14 \mathrm{~min}$ period. The position of $\mathrm{SgrA}^{*}$ is indicated by a white cross.

As a result we obtain NIR and X-ray light curves that are modulated corresponding to the random distribution of component flux densities as a function of the source component flux density $\alpha_{S}$ and the product $\theta v_{\mathrm{m}}^{5 / 4}$ (which is proportional to $B^{-4} S_{\mathrm{m}}{ }^{2}$ ). For each pair of these quantities we calculated $100 \mathrm{ran}-$ dom models and computed median values of a number of diagnostic quantities (see Fig. A.1). Here we show the contrast of the light curve calculated as defined in Sect. 2.1. The quantity $C$ is a measure of the flux modulation due to the presence of subflares during a single flare of the characteristic duration of about $100 \mathrm{~min}$. We also calculate the NIR flux density weighted magnetic field strength times the contrast of the light curve modulation. We take this quantity $F C$ (field contrast) as a measure of detectable NIR polarized flare and sub-flare structures. Finally 
Table A.1. Input parameters for the synchrotron $(A)$ and synchrotron self Compton models $(B)$. Multi component models of the temporary accretion disk around SgrA* are calculated in A.2 using component properties that are bracketed by these parameters.

\begin{tabular}{lcccc}
\hline \hline Model & $\alpha$ & $\begin{array}{c}\theta \\
\mu \text { as }\end{array}$ & $\begin{array}{c}S_{\mathrm{m}} \\
\mathrm{Jy}\end{array}$ & $\begin{array}{c}\nu_{\mathrm{m}} \\
\mathrm{GHz}\end{array}$ \\
\hline$A$ & 0.80 & 3.5 & 0.40 & 1100 \\
& 0.80 & 2.9 & 0.11 & 600 \\
$B$ & 1.30 & 6.9 & 2.40 & 1200 \\
& 1.30 & 1.5 & 0.10 & 1100 \\
\hline
\end{tabular}

we show in Fig. A.1 the NIR flux density and the corresponding synchrotron and SSC contributions. In detail the left and right hand panels in Fig. A.1 show the results for a synchrotron $(A)$ and a synchrotron self Compton model $(B)$, respectively. For both models we list input parameters in Table A.1. The top two panels $(\alpha)$ show the $\exp \left(-\chi^{2} / 2\right)$ results, the middle panels $(\beta)$ show the sub-flare contrast, and the bottom panels $(\gamma)$ show the NIR flux weighted magnetic field (see Sect. A.1), and the nearinfrared emission $(\delta)$ with red and green contour lines indicating the synchrotron and SSC contribution in that wavelength range. The white dashed line indicates the median short wavelength cutoff of the synchrotron spectrum. Significant synchrotron contribution to the total NIR flux density occurs above this line. The blue filled circle in all panels indicates the location of the minimum $\chi^{2} / 2$, i.e. maximum likelihood $\mathrm{ML}=\exp \left(-\chi^{2} / 2\right)$, and hence the location at which the model results shown in Fig. A.2 have been extracted. $\mathrm{ML}=0.5$ corresponds to a $1 \sigma$ deviation.

In order to determine the agreement between the measurements and the predicted NIR and X-ray flux density and NIR contrast $\mathrm{C}$, we perform a maximum likelihood (ML) analysis. As a ML score we use

$\log (M L)=-\chi_{S_{\mathrm{NIR}}}^{2} / 2-\chi_{S_{\mathrm{X}-\mathrm{ray}}}^{2} / 2-\chi_{S_{\mathrm{C}}}^{2} / 2$.

Here $\chi^{2}=\left(S_{\text {pred }}-S_{\text {measured }}\right)^{2} / \sigma^{2}$. For the flux densities we used the values given in Table 2 . For the sub-flare contrast $C$ we used a value of $0.6 \pm 0.3$ as derived from the May 2005 NIR data (Eckart et al. 2006b). In Fig. A.1 we show selected diagnostic diagrams for two representative synchrotron models of the flare emission of SgrA*. The models are based on the input parameters of the high and low flux density cases listed in Table A.1. These parameters represent the boundary values between which the component flux density, size and peak cutoff frequency follow a power law. We chose a spectral index of $\alpha=0.8$ for the synchrotron model (A) in order to be compatible with the constant infrared spectral index of $\alpha=0.6 \pm 0.2$ reported by Hornstein et al. (2007). For the SSC model (B) we have chosen a spectral index of $\alpha=1.3$ in order to obtain a significant contribution of SSC radiation in the NIR bands which is impossible to achieve with flatter spectral indices for the given X-ray flare brightness. The SSC X-ray flux has the same distribution as the SSC NIR flux in the NIR flux panel in Fig. A.1. For the synchrotron (A in Table A.1) and SSC (B) case it is scaled down by a factor of $\sim 1.4$ and $\sim 110$, respectively.

The QPO of the NIR data indicates that $a \geq 0.5$. We calculated the data shown in Fig. A.1 using a spin value of $a=0.5$ and an inclination of $i=70^{\circ}$ (Eckart et al. 2006b; see also Meyer et al. 2006a,b) and obtained $\chi^{2}>0.9$ and similar values for $i \geq$ $70^{\circ}$. For $a \rightarrow 1.0$ or $i \rightarrow 0^{\circ}$ the best $\chi^{2}$ value drops to 0.6 and 0.3 and its location in the panels shown in Fig. A.1 moves to the lower left.

\section{A.3. Results of the modeling}

An important result of the simulations is that the observed total NIR and X-ray flux densities can successfully be modeled simultaneously with the observed sub-flare contrast. In addition the best fits to the NIR and X-ray flux densities lie within or close to regions of high NIR flux density weighted magnetic field strength. This demonstrates that the combination of the SSC modeling and the idea of a temporary accretion disk can realistically describe the observed NIR polarized flares that occur synchronous with the 2-8 keV X-ray flares. We also find that the exponential $\alpha_{S}$ of the assumed power law distribution for the synchrotron peak flux $S_{\mathrm{m}}$ results in best model results for values around $\alpha_{S}=-1 \pm 1$. A value of $\alpha_{S}=0$ (which is included) represents scenarios in which source components cover the entire range of flux densities with an equal probability for each value rather being biased towards similarly faint or bright components. This provides high sub-flare contrast values. An exponent of $\alpha_{S}=-1$ favors lower flux density values. In the SSC model (B) high contrast is provided by the SSC contribution to the NIR spectral range also allowing for $\chi^{2}$-fits at lower flux density weighted magnetic field strengths around $30 \mathrm{G}$ rather than $60 \mathrm{G}$ as for the synchrotron model (A). These magnetic fields are comparable to the range of field strengths of the order of 0.3 Gauss to about 40 Gauss, that we obtained in our previous model calculations (Eckart et al. 2004, 2006a,b). The fields are also within the range expected for RIAF models (e.g. Markoff et al. 2001; Yuan et al. 2003, 2004) and well above the minimum value required to have the cooling time of the flare less than the duration of the flare (Yuan et al. 2003, 2004; Quataert 2003).

In Fig. A. 2 we show representative light curves and relative flux density distributions along the last stable orbit perimeter of the supermassive black hole associated with SgrA* for the synchrotron (A) and SSC (B) model.

While a single spot model, including a disk contribution, is successful in explaining the observed infrared polarized light curves, a model with two spots located at opposite sites of the temporary accretion disk may appear as an attractive solution as well. Such models are motivated by the possible formation of spiral arms within such a disk that may result in corresponding "hot spots" at the position at which the spiral arms originate and are closest to the last stable orbit. Modulation now occurs with the pattern speed rather than the orbital velocity. This is the case for the Rossby wave instability as discussed by Falanga et al. (2007).

However, a comparison between the two panels shows that for spot sizes of the order of one $R_{\mathrm{g}}$ and above such a two spot model is not very well applicable. Due to these spot sizes and due to the fact that (especially for extended spots) the boosting is a rather slow function of the spots position on its orbit, the corresponding amplification curves of an individual spot have full widths at half maxima that are in the range of 0.3 to 0.5 of a single orbital period. Such shallow amplification curves result in a rather low modulation contrast of $<0.2$ for spots of equal brightness, especially if they are located on opposite sides. If they have different brightnesses the contrast changes in favor of a single spot model as discussed in previous papers (Eckart et al. 2006b; Meyer et al. 2006a,b; Broderick \& Loeb 2006a,b). Similarly a model of equally bright spots orbiting at radii larger than the last stable orbit will result in a low modulation contrast, since the amplification is a function of the orbital velocity $\left(\propto r^{-0.5}\right)$ and therefore suffers from an additional decrease $(\Gamma(r) \rightarrow 1)$ in boosting. In summary: among orbital spot models 
Table A.2. Two sets of possible model parameters resulting from the multi-components calculations for the May 2007 and July 2004 data. We list model parameters at the beginning $t_{\text {start }}$ of the light curve sections contained in the red box in Fig. 8. The exact values of the component radii and their relative phase differences are uncertain (by about $10^{\circ}$ in $\Delta \phi$ and $30 \%$ in radius) as they are interdependent.

\begin{tabular}{ccccccc}
\hline \hline Epoch & $\begin{array}{c}\Delta \phi \text { in } \\
\text { degree }\end{array}$ & $\begin{array}{c}\text { Radius } \\
R_{\mathrm{g}}\end{array}$ & $\alpha$ & $\begin{array}{c}\text { Size } \\
\mu \text { as }\end{array}$ & $\begin{array}{c}S_{\mathrm{m}} \\
\mathrm{Jy}\end{array}$ & $\begin{array}{c}v_{\mathrm{m}} \\
\mathrm{THz}\end{array}$ \\
\hline May 2007 & 0.0 & 4.0 & 0.7 & 3.5 & 0.30 & 1.1 \\
& 0.0 & 8.4 & 0.7 & 3.5 & 0.40 & 1.1 \\
July 2004 & 0 & 4.4 & 0.8 & 3.5 & 0.40 & 1.2 \\
& -20 & 4.6 & 0.8 & 3.5 & 0.40 & 1.2 \\
& -40 & 4.8 & 0.8 & 3.5 & 0.40 & 1.2 \\
& -60 & 6.0 & 0.8 & 3.5 & 0.40 & 1.2 \\
& -80 & 6.9 & 0.8 & 3.5 & 0.40 & 1.2 \\
& -40 & 11.3 & 0.8 & 3.5 & 0.40 & 1.2 \\
& -30 & 16.2 & 0.8 & 3.5 & 0.40 & 1.2 \\
\hline & & & & & &
\end{tabular}

with spot sizes of the order of $\sim 1.5 R_{\mathrm{g}}$, the observed light curve modulations with a contrast of 0.3 to 0.6 and above, favor scenarios with a single dominant bright spot. To demonstrate that it is feasible to give a first order description of the NIR and Xray light curves with the multi-component approach, we applied the model calculations to the July 2004 data (Eckart et al. 2006a) July 2005 and May 2007 (presented here). The calculations have been performed for a spin parameter of $a=0.5$ (as a save lower limit to the spin parametrer; see Genzel et al. 2003b; Eckart et al. 2006b; Meyer et al. 2006a,b, 2007) and a disk inclination of $i=$ $70^{\circ}$. The results are summarized in Sect. A and in Table A.2.

\section{References}

Arlt, R., \& Rüdiger, G. 2001, A\&A, 374, 1035

Aschenbach, B. 2004b, A\&A, 425, 1075

Aschenbach, B., Grosso, N., Porquet, D., \& Predehl, P. 2004a, A\&A, 417, 71

Baganoff, F. K., Bautz, M. W., Brandt, W. N., et al. 2001, Nature, 413, 45

Baganoff, F. K., Maeda, Y., Morris, M., et al. 2003, ApJ, 591, 891

Balbus, S. A. 2003, ARA\&A, 41, 55

Ball, G. H., Narayan, R., \& Quataert, E. 2001, ApJ, 552, 221

Blokland, J. W. S., van der Swaluw, E., Keppens, R., \& Goedbloed, J. P. 2005, A\&A, 444, 337

Belanger, G., Terrier, R., de Jager, O. C., Goldwurm, A., \& Melia, F. 2006, J. Phys. Conf. Ser., 54, 420

Blandford, R. D., \& Königl, A. 1979, ApJ, 232, 34

Bower, G. C., Falcke, H., Sault, R. J., \& Backer, D. C. 2002, ApJ, 571, 843

Broderick, A. E., \& Loeb, A. 2006a, MNRAS, 367, 905

Broderick, A. E., \& Loeb, A. 2006b, ApJ, 636, L109

Connors, P. A., \& Stark, R. F. 1977, Nature, 269, 128

Cuadra, J., \& Nayakshin, S. 2006, J. Phys. Conf. Ser., 54, 436

Cunningham, C. T. 1975, ApJ, 202, 788

Diolaiti, E., Bendinelli, O., Bonaccini, D., et al. 2000, A\&AS, 147, 335

Eckart, A., \& Genzel, R. 1996, Nature, 383, 415

Eckart, A., Genzel, R., Hofmann, R., Sams, B. J., \& Tacconi-Garman, L. E. 1995, ApJ, 445, L23

Eckart, A., Genzel, R., Ott, T., \& Schoedel, R. 2002, MNRAS, 331, 917

Eckart, A., Baganoff, F. K., Morris, M., et al. 2004, A\&A, 427, 1

Eckart, A., Baganoff, F. K., Schödel, R., et al. 2006a, A\&A, 450, 535

Eckart, A., Schödel, R., Meyer, L., et al. 2006b, A\&A, 455, 1

Eisenhauer, F., Schödel, R., Genzel, R., et al. 2003, ApJ, 597, L121

Eisenhauer, F., Genzel, R., Alexander, T., et al. 2005, ApJ, 628, 246

Falanga, M., Melia, F., Tagger, M., Goldwurm, A., \& Belanger, M. 2007, ApJ, submitted [arXiv:astro-ph:0705.0238]

Gabuzda, D. C., \& Cawthorne, T. V. 2000, MNRAS, 319, 1056
Genzel, R., Eckart, A., Ott, T., \& Eisenhauer, F. 1997, MNRAS, 291, 219

Genzel, R., Pichon, C., Eckart, A., Gerhard, O. E., \& Ott, T. 2000, MNRAS, 317 , 348

Genzel, R., Schödel, R., Ott, T., et al. 2003a, ApJ, 594, 812

Genzel, R., Schoedel, R., Ott, T., et al. 2003b, Nature, 425, 934

Ghez, A., Klein, B. L., Morris, M., \& Becklin, E. E. 1998, ApJ, 509, 678

Ghez, A., Morris, M., Becklin, E. E., Tanner, A., \& Kremenek, T. 2000, Nature, 407, 349

Ghez, A. M., Duchêne, G., Matthews, K., et al. 2003, ApJ, 586, L127

Ghez, A. M., Wright, S. A., Matthews, K., et al. 2004, ApJ, 601, 159

Ghez, A. M., Salim, S., Hornstein, S. D., et al. 2005, ApJ, 620, 744

Gillessen, S., Eisenhaur, F., Quataert, E., et al., 2006, JPhCS, 54, 411

Goldwurm, A., Brion, E., Goldoni, P., et al. 2003, ApJ, 584, 751

Gould, R. J. 1979, A\&A, 76, 306

Greenhough, J., Chapman, S. C., Chaty, S., Dendy, R. O., \& Rowlands, G. 2001, A\&A [arXiv: astro-ph/0107074v2]

Hawley, J. F., \& Balbus, S. A. 1991, ApJ, 376, 223

Herrnstein, R. M., Zhao, J.-H., Bower, G. C., \& Goss, W. M. 2004, AJ, 127, 3399 Hirose, S., Krolik, J. H., DeVilliers, J.-P., \& Hawley, J. F. 2004, ApJ, 606, 1083

Høg, E., Fabricius, C., Makarov, V. V., et al. 2000, A\&A, 355, L27

Hornstein, S. D., Matthews, K., Ghez, A. M., et al. 2006, JPhCS, 54, 399

Hornstein, S. D., Matthews, K., Ghez, A. M., et al. 2007

[arXiv: astro-ph:0706.1782]

Liu, S., Petrosian, V., Melia, F., \& Fryer, C. L. 2006, ApJ, 648, 1020

Liu, S., Qian, L., Wu, X.-B., Fryer, C. L., \& Li, H. 2007, ApJL, submitted [arXiv: 0705.2792v2]

Markoff, S., Falcke, H., Yuan, F., \& Biermann, P. L. 2001, A\&A, 379, L13

Markoff, S., Nowak, M. A., \& Wilms, J. 2005, ApJ, 635, 1203

Markoff, S., Bower, G. C., \& Falcke, H. 2007, MNRAS, in press [arXiv: astro.ph/0702637]

Marscher, A. P. 1983, ApJ, 264, 296

Marrone, D. P., Moran, J. M., Zhao, J.-H., \& Rao, R. 2007, ApJ, 654, L57

Mauerhan, J. C., Morris, M., Walter, F., \& Baganoff, F. K. 2005, ApJ, 623, L25

Meyer, L., Eckart, A., Schödel, R., et al. 2006a, A\&A, 460, 15

Meyer, L., Schödel, R., Eckart, A., et al. 2006b, A\&A, 458, L25

Meyer, L., Eckart, A., Schödel, R., et al. 2007, A\&A, 473, 707

Narayan, R., Yi, I., \& Mehadevan, R. 1995, Nature, 374, 623

Ott, T., Eckart, A., \& Genzel, R. 1999, ApJ, 523, 248

Pessah, M. E., Chan, C.-K., \& Psaltis, D. 2007

[arXiv: astroph-0705.0352v1]

Pineault, S. 1981, ApJ, 246, 612

Pollack, L. K., Taylor, G. B., \& Zavala, R. T. 2003, ApJ, 589, 733

Quataert, E. 2003, in The central 300 parsecs of the Milky Way, ed. A. Cotera,

H. Falcke, T. R. Geballe, \& S. Markoff, Astron. Nachr. Spec. Suppl., 324, 435

Porquet, D., Predehl, P., Aschenbach, et al. 2003, A\&A, 407, L17

Reid, M. J., \& Brunthaler, A. 2004, ApJ, 616, 872

Reid, M. J., Readhead, A. C. S., Vermeulen, R. C., \& Treuhaft, R. N. 1999, ApJ, 524,816

Reid, M. J., Menten, K. M., Genzel, R., et al. 2003, ApJ, 587, 208

Rusk, R. E. 1988, Ph.D. Thesis, Universuity of Toronto, Canada

Schnittman, J. D. 2005, Ph.D. dissertation, Massachusetts Institute of Technology

Schnittman, J. D., Krolik, J. H., \& Hawley, J. F. 2006, ApJ, 651, 1031

Schödel, R., Ott, T., Genzel, R., et al. 2002, Nature, 419, 694

Schödel, R., Ott, T., Genzel, R., et al. 2003, ApJ, 596, 1015

Shakura, N. I., \& Sunyaev, R. A. 1973, A\&A, 24, 337

Tagger, M., \& Melia, F. 2006, ApJ, 636, L33

Trippe, S. 2007, private communication and presentation on a Workshop at

Schloss Ringberg (Ringberg Castle), Bavaria, Germany June 17-23, 2007:

Celebrating 15 years of precision astronomy in the Galactic Center - Hot topics and observational challenges

Trippe, S., Paumard, T., Ott, T., et al. 2007, MNRAS, 375, 764

Weisskopf, M. C., Brinkman, B., Canizares, C., et al. 2002, PASP, 114, 1

Yuan, F. 2006, JPhCS, 54, 427

Yuan, F., Markoff, S., \& Falcke, H. 2002, A\&A, 854, 854

Yuan, F., Quataert, E., \& Narayan, R. 2003, ApJ, 598, 301

Yuan, F., Quataert, E., \& Narayan, R. 2004, ApJ, 606, 894

Yusef-Zadeh, F., Roberts, D., Wardle, M., Heinke, C. O., \& Bower, G. C. 2006a, ApJ, 650, 189

Yusef-Zadeh, F., Bushouse, H., Dowell, C. D., et al. 2006b, ApJ, 644, 198

Zhao, J.-H., Young, K. H., Herrnstein, R. M., et al. 2003, ApJ, 586, L29

Zhao, J.-H., Herrnstein, R. M., Bower, G. C., Goss, W. M., \& Liu, S. M. 2004, ApJ, 603, L85 